\title{
Resource Allocation for Joint Transmitter and Receiver Energy Efficiency Maximization in Downlink OFDMA Systems
}

\author{
Qingqing Wu, Student Member, IEEE, Wen Chen, Senior Member, IEEE, Meixia Tao, Senior Member, IEEE, \\ Jun Li, Member, IEEE, Hongying Tang, and Jinsong Wu, Senior Member, IEEE
}

\begin{abstract}
This paper investigates the joint transmitter and receiver optimization for the energy efficiency (EE) in orthogonal frequency-division multiple-access (OFDMA) systems. We first establish a holistic power dissipation model for OFDMA systems, including the transmission power, signal processing power, and circuit power from both the transmitter and receiver sides, while existing works only consider the one side power consumption and also fail to capture the impact of subcarriers and users on the system EE. The EE maximization problem is formulated as a combinatorial fractional problem that is NP-hard. To make it tractable, we transform the problem of fractional form into a subtractive-form one by using the Dinkelbach transformation and then propose a joint optimization method, which leads to the asymptotically optimal solution. To reduce the computational complexity, we decompose the joint optimization into two consecutive steps, where the key idea lies in exploring the inherent fractional structure of the introduced individual $\mathrm{EE}$ and the system EE. In addition, we provide a sufficient condition under which our proposed two-step method is optimal. Numerical results demonstrate the effectiveness of proposed methods, and the effect of imperfect channel state information is also characterized.
\end{abstract}

Index Terms-Energy efficiency, OFDMA, joint transmitter and receiver optimization, resource allocation.

Manuscript received December 27, 2013; revised May 28, 2014 and September 20, 2014; accepted December 5, 2014. Date of publication December 23, 2014; date of current version February 12, 2015. This work was supported in part by the National 973 Project under Grant 2012CB316106, by NSF China under Grants 61161130529, 61328101, 61322102, and 61329101, by STCSM Science and Technology Innovation Program under Grant 13510711200, and by SEU National Key Laboratory on Mobile Communications under Grant 2013D11. The associate editor coordinating the review of this paper and approving it for publication was B. Clerckx.

Q. Wu, M. Tao, and H. Tang are with the Department of Electronic Engineering, Shanghai Jiao Tong University, Shanghai 200240, China (e-mail: wu.qq@ sjtu.edu.cn; mxtao@sjtu.edu.cn; lojordan@sjtu.edu.cn).

W. Chen is with the Department of Electronic Engineering, Shanghai Jiao Tong University, Shanghai 200240, China, and also with the School of Electronic Engineering and Automation, Guilin University of Electronic Technology, Guilin 541004, China (e-mail: wenchen@ sjtu.edu.cn).

$\mathrm{J} . \mathrm{Li}$ is with the School of Electrical and Information Engineering, The University of Sydney, Sydney, N.S.W. 2006, Australia (e-mail: jun.li1 @ sydney. edu.au).

J. Wu is with the Department of Electrical Engineering, Universidad de Chile, Santiago 412-3, Chile (e-mail: wujs@ieee.org).

Color versions of one or more of the figures in this paper are available online at http://ieeexplore.iee.org.

Digital Object Identifier 10.1109/TCOMM.2014.2385705

\section{INTRODUCTION}

$\mathbf{O}$ RTHOGONAL frequency division multiple access (OFDMA) has emerged as a promising candidate for the next generation wireless networks due to its high spectral efficiency and resistance to multipath fading [1]. Meanwhile, due to the explosive demand of data services and energy hungry multimedia wireless devices, energy efficient oriented designs are of great interests to reduce greenhouse gas emissions and lower the operational expenditure. Energy efficiency defined by bits per joule has been accepted gradually as an important metric to assess the performance of communication systems besides the system throughput [1], [2]. Therefore, investigating the energy efficiency in OFDMA systems is particularly crucial towards future wireless communication system designs.

The energy-efficient oriented designs in the OFDMA systems have attracted much attention recently [3]-[11]. The energy efficiency of the uplink scenario with flat fading channel and frequency selective channel is investigated in [3] and [4], respectively. In the downlink, the energy-efficient resource allocation with quality-of-service $(\mathrm{QoS})$ requirements is studied in [5], but the optimal solution can only be obtained through the exhaustive search for all the possible subcarrier assignments. In [7], the optimality of the OFDMA for the energy efficiency is proved under the inter-user interference in the system. The authors in [10] study the energy efficiency of a point to point OFDM system without involving the subcarrier assignment while the authors in [11] focus on energy savings in the BS side through BS cooperation in multicell OFDMA systems, where the power consumption in the receiver side and the signal processing power in terms of active subcarriers are not optimized. Therefore, the conclusions and the proposed methods therein may not be applicable considering the power consumption of the receiver side which depends on the number of active subcarriers.

In terms of system assumptions, previous works [3]-[9] may have some drawbacks for the energy efficiency evaluation. First, these works only take into consideration of the power consumption from the BS side. Practically, the concept of energy efficiency should involve the system overall throughput and the system overall power consumption and it has been pointed out that techniques adopted to improve the energy efficiency of one end of the communication system may adversely affect the energy efficiency of the other end [12], [13]. In addition, due to the limited capacity of batteries, we should also carefully 
manage the power consumption in the user terminal side which is also a crucial issue associated with the user experience [14][16]. Meanwhile, as cellular communication networks evolve, the cell coverage regions continue to shrink in size in order to meet the high data demands or various kinds of QoS, such as micro cells and femto cells, where the power consumption of the user terminal is no longer negligible and it is in fact comparable with the BS [13]-[16]. That is, the joint transmitter and receiver optimization from a holistic perspective is required when evaluating the energy efficiency of a communication system. Second, most of these works either ignore the signal processing or assume that it is a constant regardless of the bandwidth. However, according to the system power dissipation concluded from industrial circle recently, the signal processing power linearly increases with the bandwidth or the number of active subcarriers [17], [18]. Therefore, the constant circuitlevel modeling in [3]-[9] is failed to capture the characteristics of energy efficiency enhancement with respect to subcarriers and users.

In this paper, we investigate the system energy efficiency considering all the above issues. Specifically, we focus on the resource allocation for joint transmitter and receiver energy efficiency maximization problem of OFDMA systems. We first establish a general power consumption model in multiuser OFDM systems by taking account of the transmission power from the base station side, the signal processing power and the fixed circuit power from both sides. Then, we propose a joint optimization method to iteratively find the optimal solution for the energy efficiency maximization problem based on the Dinkelbach transformation which has been widely used in energy efficiency optimization area [10], [11].

In order to reduce the computational complexity, we propose a low complexity two-step method based on the relationship of the introduced individual energy efficiency of the subcarrieruser pattern and the system energy efficiency. Specifically, we first design an efficient subcarrier assignment scheme according to the individual energy efficiency of the subcarrier-user pattern. For the power allocation, a linear complexity scheme is developed by exploring the fractional structure of the system energy efficiency. In addition, we also provide a sufficient condition under which our proposed low complexity method is optimal. From simulation results we can see the trade-off between the spectral efficiency and the energy efficiency. It is interesting to note that the energy efficiency of the proposed method outperforms the conventional throughput maximization method, even in the low power region due to the optimization for the number of active subcarriers. Moreover, it is found that exploiting more user diversity is not always beneficial from the perspective of energy efficiency.

The remainder of this paper is organized as follows. In Section II, we establish the power consumption model in OFDMA systems and formulate the energy efficiency maximization problem. In Section III, a joint optimization method is proposed to iteratively obtain the asymptotically optimal SU pairing and power allocation. In Section IV, we design a two-step method to obtain the near-optimal solution with low complexity. Section $\mathrm{V}$ provides the simulation results and the paper is concluded in Section VI.

\section{System Description And Problem Formulation}

In this section, we first introduce the system model of OFDMA systems and then formulate the joint transmitter and receiver energy efficiency maximization problem.

\section{A. System Description}

Consider a downlink OFDMA system in a single cell with one BS and $K$ users which are all equipped with one antenna. The total bandwidth $W$ is equally divided into $N$ orthogonal subcarriers, each with a bandwidth of $B=W / N$. The channel is modeled to have both the large-scale attenuation and the smallscale fading. Specifically, the large-scale attenuation includes distance-dependent path loss and shadowing. The small-scale fading is assumed to be frequency selective and independent among different users. The channel coefficients are assumed to keep unchanged within each scheduling interval, which consists of multiple OFDM symbols. We assume that perfect channel state information at the transmitter (CSIT) is available [19][21] and the effects of imperfect CSIT will be demonstrated in the simulation. In practice, the CSIT can be typically collected by estimating it at each user terminal and sending it to the BS via a feedback channel a frequency division duplex system, or through channel estimation of the uplink in a time division duplex system [11], [22]. We also assume that each subcarrier is exclusively assigned to at most one user in each scheduling interval, in order to avoid the interference among different users. Each user, on the other hand, can occupy more than one subcarrier.

Denote $p_{i, j}$ and $g_{i, j}$ as the allocated power and the channel gain for user $j$ on subcarrier $i$, respectively. Then, the maximum achievable data rate of user $j$ on subcarrier $i$, denoted as $r_{i, j}$, is given by

$$
r_{i, j}=B \log _{2}\left(1+\frac{p_{i, j} g_{i, j}}{N_{0} B}\right)
$$

where $N_{0}$ is the spectral density of the additive white Gaussian noise. The system total data rate is defined as the weighted sum rate of all the users, where the weight of user $j$ is denoted as $\omega_{j}$. Note that the weights are introduced to guarantee fairness among the users. Specifically, the controller can vary the weights of different users to offer different priorities and enforce certain concepts of fairness [19], [21]. Therefore, the overall system data rate is given by

$$
R_{t o t}=\sum_{j=1}^{K} \omega_{j} \sum_{i=1}^{N} \rho_{i, j} r_{i, j}
$$

where the binary indicator $\rho_{i, j} \in\{0,1\}$ indicates that subcarrier $i$ is assigned to user $j$ if $\rho_{i, j}=1$, otherwise $\rho_{i, j}=0$.

\section{B. Power Consumption Model of the Whole System}

The power consumption of the BS includes the over-the-air transmission power, the signal processing power, and a fixed 
circuit power $P_{b}$ [17], [23], [24]. Mathematically, it can be expressed as

$$
P_{t}=\sum_{i=1}^{N} \sum_{j=1}^{K} \rho_{i, j}\left(\frac{p_{i, j}}{\xi}+P_{s 0}\right)+P_{b},
$$

where $P_{s 0}$ denotes the signal processing power per subcarrier [17] and $\xi \in(0,1)$ is a constant which accounts for efficiency of the amplifier [1], [8], [11], [25]-[28]. Even though in practical scenario there may not be the strict linearity, it has been shown in [29] that a linear abstraction model for the amplifier is effective enough to characterize the reality. In addition, it is important to mention from the energy efficiency perspective that the subcarrier assignment variable $\rho_{i, j}$ must be zero when the corresponding power allocation variable $p_{i, j}$ is zero. Otherwise, there will be a waste of signal processing power $P_{s 0}$.

The power consumption of each user includes the signal processing power which linearly scales with the number of active subcarriers and a fixed circuit power [17], [23], [24], and it is given by

$$
P_{r j}=\sum_{i=1}^{N} \rho_{i, j} P_{s j}+P_{u j}
$$

where $P_{s j}$ and $P_{u j}$ are the per-subcarrier signal processing power and the fixed circuit power of user $j$, respectively. Considering that different users may employ different types of mobile terminals, $P_{s j}$ and $P_{u j}$ can be different for different $j$. Therefore, the overall system power consumption including the BS side and the user side is given by

$$
P_{t o t}=P_{t}+\sum_{j=1}^{K} P_{r j} .
$$

After some manipulations, $P_{t o t}$ can be rewritten as

$$
P_{\text {tot }}=\sum_{i=1}^{N} \sum_{j=1}^{K} \rho_{i, j} \underbrace{\left(\frac{p_{i, j}}{\xi}+P_{s 0}+P_{s j}\right)}_{P_{i, j}}+\underbrace{\sum_{j=1}^{K} P_{u j}+P_{b}}_{P_{0}} .
$$

Note that the physical interpretation of $P_{i, j}$ is the power consumed by the overall system (i.e., including both the transmitter and the receiver) if subcarrier $i$ is assigned to user $j . P_{0}$ denotes the constant circuit power of the whole system which scales with the total number of users in the system. Although this paper only focuses on the downlink scenario, the power consumption model established above is also applicable to evaluating the system energy efficiency of the uplink scenario simply by changing the user as the transmitter side and the BS as the receiver side.

\section{Problem Formulation}

Energy efficiency has become an increasingly important metric to assess the performance of communication systems. In this work, it is defined as the overall system data rate $R_{t o t}$ over the overall system power consumption $P_{t o t}$, i.e.

$$
E E=\frac{R_{t o t}}{P_{t o t}} .
$$

Our goal is to optimize the subcarrier assignment and the power allocation for energy efficiency maximization subject to a peak transmit power constraint at the BS and a minimum system sum-rate requirement. Mathematically, we can formulate the energy efficiency optimization problem as

$$
\begin{array}{ll}
\max _{\rho, p} & \frac{\sum_{j=1}^{K} \omega_{j} \sum_{i=1}^{N} \rho_{i, j} r_{i, j}}{\sum_{i=1}^{N} \sum_{j=1}^{K} \rho_{i, j} P_{i, j}+P_{0}} \\
\text { s.t. } & \sum_{i=1}^{N} \sum_{j=1}^{K} \rho_{i, j} p_{i, j} \leqslant P_{\max }, \\
& \sum_{i=1}^{N} \sum_{j=1}^{K} \rho_{i, j} r_{i, j} \geqslant R_{\min }, \\
& p_{i, j} \geqslant 0, \quad \forall i, j, \\
& \sum_{j=1}^{K} \rho_{i, j} \leqslant 1, \forall i, \rho_{i, j} \in\{0,1\}, \forall i, j,
\end{array}
$$

where $\rho \triangleq\left\{\rho_{i, j} \mid i=1,2, \ldots N ; j=1,2, \ldots, K\right\}$ and $p \triangleq\left\{p_{i, j} \mid i=\right.$ $1,2 \ldots N ; j=1,2 \ldots, K\}$.

In problem (8), constraint $(8 \mathrm{~b})$ is the maximum transmit power constraint of the BS. This maximum power $P_{\max }$ not only control the out-cell interference, but also restrict the power amplifier to work in the linear region which corresponds to the constant amplifier efficiency $\xi$. Constraint (8c) is the minimum system data rate requirement where $R_{\min }$ can be varied to trade off the energy efficiency and the spectral efficiency, and thereby guarantee the system quality of service in a certain extent. Constraints (8d) and (8e) are to guarantee the feasible sets of $p$ and $\rho$ in the considered system, respectively.

It is very challenging to solve problem (8) due to the existence of the binary variables $\rho_{i, j}$ and the non-convex fractional structure of the objective function. In fact, unlike the conclusions in [5]-[7], even if the total transmission power is fixed, it is still not equivalent to the conventional throughput maximization problem since the number of active subcarriers associated with the signal processing power also needs to be optimized, which would further complicate the analysis of the problem.

\section{JOINT OPTIMIZATION BASED ON DinKelbaCh Method}

In this section, we propose an asymptotically optimal subcarrier assignment and power allocation method by using Dinkelbach method together with dual decomposition method.

\section{A. Problem Transformation}

Dinkelbach method is known as an iterative method to solve the fractional programming problem with a sequence of parameterized programming problems. Its notion is to transform a fractional-form problem into a subtractive-form problem. The equivalent transformation statement is summarized in the following theorem [26], [30]. 
Theorem 1: Let $S=\left\{(\rho, p) \mid \sum_{j=1}^{K} \rho_{i, j} \leqslant 1, \forall i ; p_{i, j} \geqslant 0, \rho_{i, j} \in\right.$ $\{0,1\}, \forall i, j\}$. There is

$$
q^{*}=\max _{(\rho, p) \in S} \frac{R_{t o l}(\rho, p)}{P_{t o l}(\rho, p)}
$$

if and only if,

$$
U\left(q^{*}\right)=\max _{(\rho, p) \in S}\left\{R_{t o l}(\rho, p)-q^{*} P_{t o l}(\rho, p)\right\}=0 .
$$

In Theorem $1, q^{*}$ is the optimal system energy efficiency to be determined. Dinkelbach [30] provides a method to iteratively update $q$. In each iteration, it solves a subtractive-form maximization problem (10) with a given $q$ and then judge whether it converges. If not, update $q$ and repeat the maximization problem (10) until it converges or reaches the maximal iterations. For further details and the proof of convergence, the readers can refer to [26], [30].

Define $V(\rho, p, q) \triangleq R_{t o l}(\rho, p)-q P_{t o l}(\rho, p)$. After the equivalent transformation of Theorem 1, in order to solve the optimization problem (8), we only need to solve the following maximization problem for given $q$,

$$
\begin{aligned}
\max _{(\rho, p)} & V(\rho, p, q)=R_{\text {tol }}(\rho, p)-q P_{\text {tol }}(\rho, p) \\
\text { s.t. } & \sum_{i=1}^{N} \sum_{j=1}^{K} \rho_{i, j} p_{i, j} \leqslant P_{\max }, \\
& \sum_{i=1}^{N} \sum_{j=1}^{K} \rho_{i, j} r_{i, j} \geqslant R_{\min }, \\
& p_{i, j} \geqslant 0, \forall i, j, \\
& \sum_{j=1}^{K} \rho_{i, j} \leqslant 1, \forall i, \rho_{i, j} \in\{0,1\}, \forall i, j .
\end{aligned}
$$

Due to binary variables $\rho_{i, j}$, problem (11) is still a mixed combinatorial optimization problem. In general, the duality gap of problem (11) and its dual problem is not zero because of its non-convexity [31]. However, it has been proved in [11], [32] that for practical multicarrier systems with a large number of subcarriers, the so-called time-sharing condition is satisfied, which implies a zero duality gap between the primal problem and its dual problem. Therefore, the optimal solution of the dual problem is also the solution of the primal problem. In the following subsections, we shall also apply the results from [11] to solve our problem (11) in the dual domain.

\section{B. Optimal Subcarrier Assignment and Power Allocation at a Given Dual Point}

The Lagrangian function of problem (11) is

$$
\begin{aligned}
& \mathcal{L}(\rho, p, \lambda, \mu) \\
& =\sum_{i=1}^{N} \sum_{j=1}^{K} \omega_{j} \rho_{i, j} r_{i, j}+\lambda\left(P_{\max }-\sum_{i=1}^{N} \sum_{j=1}^{K} \rho_{i, j} p_{i, j}\right)
\end{aligned}
$$

$$
\begin{aligned}
& -q\left(\sum_{i=1}^{N} \sum_{j=1}^{K} \rho_{i, j} P_{i, j}+P_{0}\right)+\mu\left(\sum_{i=1}^{N} \sum_{j=1}^{K} \rho_{i, j} r_{i, j}-R_{\min }\right) \\
& =\sum_{i=1}^{N} \sum_{j=1}^{K} \rho_{i, j} X_{i, j}\left(p_{i, j}\right)+\lambda P_{\max }-\mu R_{\min }-q P_{0},
\end{aligned}
$$

where

$$
X_{i, j}\left(p_{i, j}\right)=\left(\omega_{j}+\mu\right) r_{i, j}-q P_{i, j}-\lambda p_{i, j} .
$$

In the above equation, $\lambda$ and $\mu$ are the Lagrange multipliers corresponding to the maximum transmit power constraint (11b) and the minimum system data rate constraint (11c), respectively. Note that the boundary constraints with respect to $\rho_{i, j}$ and $p_{i, j}$ will be absorbed into the optimal solution in the following. Then the associated dual function of problem (11) is

$$
g(\lambda, \mu)=\max _{\rho, p} \mathcal{L}(\rho, p, \lambda, \mu), \text { s.t. }(11 \mathrm{~d}),(11 \mathrm{e}) .
$$

From (12) and (14), we can observe that the coupling constraints (11b) and (11c) have been removed and $g(\lambda, \mu)$ can be decomposed into $N$ subproblems which can be solved independently with given dual variables $\lambda$ and $\mu$. In addition, due to the decoupling of $\rho$ and $p$ in constraints, we can first maximize $\mathcal{L}(\rho, p, \lambda, \mu)$ over the power allocation variables $p$. Then according to the derived power allocation results, we obtain the optimal subcarrier assignment indicator $\rho$.

In order to maximize $\mathcal{L}(\rho, p, \lambda, \mu)$ for given $\lambda$ and $\mu$, we should maximize each $X_{i, j}\left(p_{i, j}\right)$ for each subcarrier. It is easy to verify that $X_{i, j}\left(p_{i, j}\right)$ is concave with respect to $p_{i, j}$, where the stationary point is also the optimal point. By setting the derivative of $X_{i, j}\left(p_{i, j}\right)$ with respect to $p_{i, j}$ to zero, the optimal power allocation of user $j$ on subcarrier $i$ is

$$
p_{i, j}^{*}=\left[\frac{B \xi\left(\omega_{j}+\mu\right)}{(q+\lambda \xi) \ln 2}-\frac{B N_{0}}{g_{i, j}}\right]^{+},
$$

where $[x]^{+}$denotes $\max (x, 0)$. The above power allocation result looks like the classical water filling policy. Here, the water-level depends not only on the dual variables but also on the individual weight $w_{j}$ of user $j$, thereby resulting in the multi-level water-filling policy. Moreover, since $q$ increases as the iteration goes according to the Dingkebach method, the water-level $B \xi\left(\omega_{j}+\mu\right) /(q+\lambda \xi) \ln 2$ will decrease on the contrary. This implies to use less power to achieve higher energy efficiency. In addition, from (11a), $q$ can also be interpreted as the penalty for power in accord with the Lagrangian multiplier associated with the power constraint in the conventional throughput maximization problem.

Substituting $p_{i, j}^{*}$ back into (13), we can obtain the optimal value of $X_{i, j}\left(p_{i, j}\right)$ which is denoted as $X_{i, j}^{*}\left(p_{i, j}^{*}\right)$. Here, we should note that $X_{i, j}^{*}\left(p_{i, j}^{*}\right)$ is not guaranteed to be positive even if $p_{i, j}^{*}>0$, since it depends on the given values of $q$ and $g_{i, j}$. As we can see in the following, the positive uncertainty of 
$X_{i, j}^{*}\left(p_{i, j}^{*}\right)$ will make a critical impact for the optimal assignment indicator. After obtaining the optimal power allocation $p^{*}$, we next determine the optimal subcarrier assignment indicator $\rho^{*}$. From (14), we have

$$
\mathcal{L}\left(\rho, p^{*}, \lambda, \mu\right)=\sum_{i=1}^{N} \sum_{j=1}^{K} \rho_{i, j} X_{i, j}^{*}+\lambda P_{\max }-\mu R_{\min }-q P_{0}
$$

As we know that $X_{i, j}^{*}$ can be regarded as the marginal benefit of assigning subcarrier $i$ to user $j$. Note that the constraints on the assignment indicator for different subcarriers are independent in (11d). Then the problem (11) can be decomposed into $N$ subproblems in parallel, which means that we can select the optimal user for each subcarrier independently. Recall the fact that the subcarrier can only be exclusively assigned to at most one user. Then there is only at most one $\rho_{i, j}$ having the binary value of 1 . Therefore, the optimal subcarrier assignment indicator is given by, for all $i$,

$$
\rho_{i, j}^{*}= \begin{cases}1, & j=\arg \max _{j^{\prime}=1, \ldots, K} X_{i, j^{\prime}}^{*} \text { and } X_{i, j^{\prime}}^{*}>0, \\ 0, & \text { otherwise. }\end{cases}
$$

From (15) and (17), we can see that if $X_{i, j^{\prime}}^{*} \leqslant 0$ for $p_{i, j^{\prime}}^{*}>0$, it means that assigning subcarrier $i$ to its best user $j^{\prime}$ still cannot result in the positive marginal benefit. In other words, this subcarrier should not be activated from the perspective of energy efficiency.

\section{Dual Problem Optimization}

After computing $\rho^{*}$ and $p^{*}$ with given $\lambda$ and $\mu$ in Subsection-B, we now solve the standard dual optimization problem which is

$$
\begin{array}{ll}
\min _{\lambda, \mu} & g(\lambda, \mu) \\
\text { s.t. } & \lambda \geqslant 0, \mu \geqslant 0 .
\end{array}
$$

Since the dual problem is always a convex optimization problem by definition, the commonly used ellipsoid method and subgradient method can be employed to update $\lambda$ and $\mu$ toward the optimal solution with global convergence. Here, we adopt the ellipsoid since the subgradient method needs to determine the step size which has a critical impact on the performance and the convergence. According to (14), the subgradient of the dual function is given as follows

$$
\begin{aligned}
& \Delta \lambda=P_{\max }-\sum_{i=1}^{N} \sum_{j=1}^{K} \rho_{i, j}^{*} p_{i, j}^{*}, \\
& \Delta \mu=\sum_{i=1}^{N} \sum_{j=1}^{K} \rho_{i, j}^{*} B \log _{2}\left(1+\frac{p_{i, j}^{*} g_{i, j}}{N_{0} B}\right)-R_{\min } .
\end{aligned}
$$

The details of a suitable choice of the initial ellipsoid, the updating of the ellipsoid, and the stopping criterion can be found in [22], [31].

\section{Power Refinement and Overall Resource Allocation Algorithm}

Having obtained the optimal dual variables $\lambda$ and $\mu$, we now determine the optimal primal variables to the primal problem (11). If the optimal subcarrier assignment $\rho^{*}$ and power allocation $p^{*}$ at the optimal dual point $\left(\lambda^{*}, \mu^{*}\right)$ satisfy the power constraint, then duality gap is zero and the solution is globally optimal. Otherwise, if the power allocation $p^{*}$ exceed the maximum transmit power, then the duality gap is non-zero and we need to refine the power allocation and restrain it into the feasible region. Specifically, the primal subcarrier assignment is assumed to be $\rho^{*}$, and the refined power allocation $p^{*}$ is then found by classical waterfilling policy over all the active subcarriers indicated by $\rho^{*}$.

Finally, combining all the above subsections, the overall procedure to solve the original problem (8) is outlined in Algorithm 1.

Algorithm 1 Joint transmitter and receiver energy efficiency optimization

1: Initialization: Given the initial $q=0, n=0$, the maximal tolerance $\varepsilon$, and the maximal iterations $L_{\max }$;

2: repeat

3: initialize $\lambda, \mu$, and an initial ellipsoid;

4: repeat

5: $\quad$ Compute $p_{i, j}^{*}$ and $X_{i, j}^{*}$ using (15) and (13) with given $q, \lambda$, and $\mu$;

6: $\quad$ Determine the optimal assignment indicator $\rho_{i, j}^{*}$ by (17) for all subcarriers;

7: $\quad$ Update dual variables $\lambda, \mu$, and the ellipsoid with subgradients in (19) and (20);

8: until $\lambda$ and $\mu$ converge

9: Refine the power allocation;

10: $\quad$ if $V\left(\rho^{*}, p^{*}, q\right)>\varepsilon$ and $n<L_{\max }$

11: $n=n+1$;

12: $\quad$ Update $q=\frac{R_{t o l}\left(\rho^{*}, p^{*}\right)}{P_{t o l}\left(\rho^{*}, p^{*}\right)}$

13: else Convergence

14: $\quad p^{o p t}=p^{*}$

15: $\quad \rho^{o p t}=\rho^{*}$;

16: $\quad E E^{o p t}=q^{*}$;

17: return;

18: end

19: end

\section{LOW COMPlexity ENERGY-EFFICIENT DESIGN}

Since the joint optimization method needs to iteratively converge to the optimal solution, its high computational complexity would lead to additional energy cost which may degrade the expectation of the energy-efficient design. In this section, we develop a low-complexity two-step method to obtain a near optimal solution by addressing the system energy efficiency directly from a fractional-form perspective. The key idea lies in the relationship between the individual energy efficiency $e e_{i, j}$ and the system energy efficiency $E E$. In the next, we first 
introduce the individual energy efficiency of one subcarrieruser pattern.

\section{A. Individual Energy Efficiency of One Subcarrier-User Pattern}

Assume that subcarrier $i$ is assigned to user $j$. Define the individual energy efficiency (iEE) of this subcarrier-user pattern as

$$
e e_{i, j}=\frac{\omega_{j} r_{i, j}}{P_{i, j}}=\frac{\omega_{j} B \log _{2}\left(1+\frac{p_{i, j} g_{i, j}}{N_{0} B}\right)}{\frac{p_{i, j}}{\xi}+P_{s 0}+P_{s j}},
$$

where $P_{i j}$ is defined in (6). From (21), we can observe that the individual energy efficiency $e e_{i, j}$ increases with the channel gain $g_{i, j}$ and the user weight $\omega_{j}$, and decreases with the signal processing power $P_{s 0}$ and $P_{s j}$, respectively. In addition, it is easy to show that $e e_{i, j}$ is a strictly quasiconcave function of $p_{i, j}$. Although the stationary point is not necessarily the optimal point for a strictly quasiconcave function [31], this fractional type function has been proved in [33] to have the stationary point which is also the optimal point. Due to this property, we set the partial derivative of $e e_{i, j}$ with respect to $p_{i, j}$ to zero, i.e.,

$$
\frac{\partial e e_{i, j}}{\partial p_{i, j}}=\frac{\frac{\omega_{j} B g_{i, j}}{\left(N_{0} B+p_{i, j} g_{i, j}\right) \ln 2} P_{i, j}-\frac{\omega_{j}}{\xi} r_{i, j}}{\left(\frac{p_{i, j}}{\xi}+P_{s 0}+P_{s j}\right)^{2}}=0 .
$$

After some simple manipulations, we can get the optimal power allocation $p_{i, j}^{*}$ and the optimal energy efficiency $e e_{i, j}^{*}$ as follows

$$
e e_{i, j}^{*}=\frac{\xi \omega_{j} B g_{i, j}}{\left(B N_{0}+p_{i, j}^{*} g_{i, j}\right) \ln 2} .
$$

Based on (21) and (23), numerical values of $e e_{i, j}^{*}$ and $p_{i, j}^{*}$ can be easily obtained. In the following, we will show that this introduced individual energy efficiency will play an important role in the algorithm design.

\section{B. Efficient Subcarrier Assignment}

In this subsection, we first investigate a special case of the optimal subcarrier assignment, which will provide valuable insights for the proposed heuristic scheme design. By separating subcarrier $m$ with the other subcarriers, the system energy efficiency can be expressed as

$$
E E=\frac{\sum_{i \neq m} \sum_{j=1}^{K} \rho_{i, j} \omega_{j} r_{i, j}+\sum_{j=1}^{K} \rho_{m, j} \omega_{j} r_{m, j}}{\left(\sum_{i \neq m} \sum_{j=1}^{K} \rho_{i, j} P_{i, j}+P_{0}\right)+\sum_{j=1}^{K} \rho_{m, j} P_{m, j}} .
$$

From (24) we can see that if the allocated power $p_{m, j}$ on subcarrier $m$ is zero for all $j$, then subcarrier $m$ makes no contribution to the system energy efficiency, since the pattern rate $r_{m, j}$ are zeros for all $j$. On the other hand, if $p_{m, j}$ is nonzero for some $j$, we should determine which user should occupy subcarrier $m$. Assume that subcarrier $m$ is assigned to user $k$, i.e., $\rho_{m, k}=1$, and $\rho_{m, j}=0$ for $j \neq k$. Substituting the expression of rate and power for subcarrier $m$ into (24), we have

$$
E E=\frac{\sum_{i \neq m} \sum_{j=1}^{K} \rho_{i, j} \omega_{j} r_{i, j}+\omega_{k} B \log _{2}\left(1+\frac{p_{m, k} g_{m, k}}{N_{0} B}\right)}{\left(\sum_{i \neq m} \sum_{j=1}^{K} \rho_{i, j} P_{i, j}+P_{0}\right)+\left(\frac{p_{m, k}}{\xi}+P_{s 0}+P_{s k}\right)} .
$$

Based on the observation of (25), we get the following theorem.

Theorem 2: For subcarrier $m$, if there is a certain user $k$ with the following property:

$$
\begin{aligned}
\omega_{k} & =\max _{j=1, \ldots, K} \omega_{j}, \\
g_{m, k} & =\max _{j=1, \ldots, K} h_{m, j}, \\
P_{s k} & =\min _{j=1, \ldots, K} P_{s j},
\end{aligned}
$$

then user $k$ is the optimal user occupying subcarrier $m$ in maximizing $E E$.

Proof: Please see Appendix A.

Theorem 2 indicates that if a user can contribute the highest data rate with the lowest power consumption among all the users on subcarrier $m$, then we should assign subcarrier $m$ to this user regardless of the power allocation. In practice, there are chances that on certain subcarriers, no user will have the property in Theorem 2. Nevertheless, motivated by Theorem 2, we propose to adopt the maximal individual energy efficiency (iEE) $e e_{i, j}^{*}$ as the metric to select the user for each subcarrier. Then the proposed heuristic subcarrier assignment policy is

$$
\rho_{i, j}= \begin{cases}1, & j=\arg \max _{j^{\prime}=1, \ldots, K} e e_{i, j^{\prime}}^{*}, \\ 0, & \text { otherwise, } \forall i,\end{cases}
$$

where $e e_{i, j}^{*}$ is defined in (23).

Note that this scheme is not sufficient for the optimality of $E E$ since it does not consider the optimal power allocation and the assignment of other subcarriers. However, on the same subcarrier, the only different items among users are the weight $\omega_{j}$, the channel gain $g_{i, j}$ and signal processing power $P_{s j}$. Clearly, the maximum individual energy efficiency $e e_{i, j}^{*}$ of a subcarrieruser pattern involves all of them. From Section IV-A, we know that higher iEE implies that the user has larger $\omega_{j}$ or higher $g_{i, j}$ or lower $P_{s j}$ in some sense. Meanwhile, from Theorem 2, the user $j$ with the larger $\omega_{j}$, higher $g_{i, j}$ and lower $P_{s j}$ is more preferable to occupy the subcarrier $i$ in order to achieve the highest energy efficiency. Therefore, it is rational to adopt this iEE based scheme for the subcarrier assignment. Simulation results in Section V will demonstrate the effectiveness of this subcarrier assignment scheme.

\section{Efficient Power Allocation}

In this subsection, we investigate the power allocation with given subcarrier assignment policy $\rho_{i, j}$ in Section III-B. Since each subcarrier $i$ has been assigned to a unique user $j$ by (27), the considered system is then simplified to a parallel-channel system with $N$ subcarriers. For notation simplicity, the user index $j$ is dropped in the subsequent discussion and denote $P_{c i} \triangleq P_{s 0}+P_{s j}$. Furthermore, if the power allocation $p_{i}$ is zero, 
the signal processing power associated with subcarrier $i$ should also be zero. Therefore, we need to introduce the set of active subcarriers as follows.

Denote $R$ as the set of subcarriers that are allocated with positive powers, i.e., $R=\left\{i \mid p_{i}>0\right\}$. Then, problem (8) is simplified into the following problem,

$$
\begin{array}{ll}
\max _{p} & E E=\frac{\sum_{i \in R} \omega_{i} B \log _{2}\left(1+\frac{p_{i} g_{i}}{N_{0} B}\right)}{\sum_{i \in R}\left(\frac{p_{i}}{\xi}+P_{c i}\right)+P_{0}} \\
\text { s.t. } & \sum_{i \in R} p_{i} \leqslant P_{\max }, \\
& \sum_{i \in R} B \log _{2}\left(1+\frac{p_{i} g_{i}}{N_{0} B}\right) \geqslant R_{\min } .
\end{array}
$$

According to the classical water-filling policy, constraint (28c) is actually requiring a minimum total transmit power so that to guarantee the spectral efficiency. Denote $P_{\min }$ as the total transmit power required in achieving the minimum system data rate $R_{\max }$, i.e.,

$$
\sum_{i \in R} B \log _{2}\left(1+\frac{p_{i} g_{i}}{N_{0} B}\right)=R_{\min } \text { and } \sum_{i \in R} p_{i}=P_{\min } .
$$

Based on the above, we can further transform problem (28) into the following,

$$
\begin{array}{ll}
\max _{p} & E E=\frac{\sum_{i \in R} \omega_{i} B \log _{2}\left(1+\frac{p_{i} g_{i}}{N_{0} B}\right)}{\sum_{i \in R}\left(\frac{p_{i}}{\xi}+P_{c i}\right)+P_{0}} \\
\text { s.t. } & P_{\min } \leqslant \sum_{i \in R} p_{i} \leqslant P_{\max } .
\end{array}
$$

Given the active subcarrier set $R, \sum_{i \in R} P_{c i}+P_{0}$ in the denominator is fixed and problem (30) is a standard quasiconcave optimization problem [31]. The optimal solution can be easily obtained by the conventional bisection based water-filling method in [10], [34]. In the next, we first investigate the case without the constraint (30b) to obtain the optimal subcarrier set $R$, which motivates the low complexity scheme design.

Denote $R^{\text {opt }}$ as the optimal set of the problem (30) without the constraint (30b). Our task is then reduced to finding $R^{o p t}$ in which the allocated power for each subcarrier is positive. However, the complexity for searching the optimal set $R^{o p t}$ is $2^{N}-1$ since there are $N$ subcarriers in the system. In the following, we propose an $i E E$ ordering based scheme which is optimal for the power allocation only with a linear complexity.

The key idea of this scheme is based on exploiting the inherent fractional property of $e e_{i}^{*}$ and $E E$. We first sort all subcarriers by $e e_{i}^{*}$ in descending order according to (23), i.e., $e e_{1}^{*} \geqslant e e_{2}^{*} \geqslant \ldots \geqslant e e_{N}^{*}$. Then, we add each subcarrier to set $R$ successively according to the order. In the $L$ th round, we should determine whether the $L$ th subcarrier should be added to the set $R$. Let $E E_{L}=\left(\sum_{l=1}^{L} r_{l}\right) /\left(\sum_{l=1}^{L} P_{l}+P_{0}\right)$ and $E E_{L}^{*}$ is denoted as its optimal value. We have the following theorem.
Theorem 3: 1) If $E E_{L-1}^{*} \leqslant e e_{L}^{*}$, then there must be $E E_{L-1}^{*} \leqslant$ $E E_{L}^{*} \leqslant e e_{L}^{*}$ and the $L$ th subcarrier should be added to the set $R$; 2) If $E E_{L-1}^{*}>e e_{L}^{*}$, then there must be $E E_{L-1}^{*}>E E_{L}^{*}>e e_{L}^{*}$ and the $L$ th subcarrier should not be added to the set $R$.

Proof: Please see Appendix B.

Theorem 3 implies that in the $L$ th round the comparison result of $E E_{L-1}^{*}$ and $e e_{L}^{*}$ is sufficient to determine whether the $L$ th subcarrier should be added to set $R$ in the optimal solution. Based on Theorem 3 and the ordering property, we further have the following corollary.

Corollary: When $E E_{L-1}^{*}>e e_{L}^{*}$, the first $L-1$ subcarriers are allocated with positive powers and compose the optimal set $R^{o p t}$ and $E E_{L-1}^{*}$ is the highest system energy efficiency for given $\rho^{*}$.

Proof: Please see Appendix C.

Corollary 1 implies that subcarriers behind the $L$ th subcarrier cannot be added to set $R$ either, which means that the set including the first $L-1$ subcarriers is the optimal set $R^{\text {opt }}$ and $E E_{L-1}^{*}$ is the optimal system energy efficiency for given $\rho$ in Section III-B.

After considering the case study without constraints, we now proceed to address the case with the maximum transmit power and the minimum system data rate constraints, i.e., problem (30). Similarly, we firstly sort all subcarriers by their iEE $e e_{i}^{*}$ in descending order according to (23), i.e., $e e_{1}^{*} \geqslant e e_{2}^{*} \geqslant \ldots \geqslant e e_{N}^{*}$. Then we add each subcarrier to the set $R$ successively according to the order. In the $(L-1)$ th round, we solve problem (30) and denote its optimal value as $E E_{L-1}^{*}$. Then following the procedure of Theorem 3, we compare $E E_{L-1}^{*}$ with $e e_{L}^{*}$.

- If $E E_{L-1}^{*} \leqslant e e_{L}^{*}$, unlike the conclusion in Theorem 3, adding the $L$ th subcarrier is not certain to improve the system energy efficiency due to the restriction of $P_{\max }$ (inequality (a) in (32) may not hold). Thus, we need to compare $E E_{L}^{*}$ with $E E_{L-1}^{*}$. If there further exists $E E_{L-1}^{*} \leqslant$ $E E_{L}^{*}$, this implies that adding the $L$ th subcarrier can indeed improve the system energy efficiency, otherwise, it cannot be added to the scheduling set. Then, we jump over this subcarrier and go to judge the next subcarrier.

- If $E E_{L-1}^{*}>e e_{L}^{*}$, the statement $E E_{L-1}^{*}>E E_{L}^{*}$ in Theorem 3 also may not hold true due to the restriction of $P_{\min }$. Similarly, by the previous conclusion, we need to compare $E E_{L}^{*}$ with $E E_{L-1}^{*}$. If there further exists $E E_{L-1}^{*}>E E_{L}^{*}$, this implies that adding the $L$ th subcarrier cannot improve the system energy efficiency, otherwise, it should be added to the scheduling set. Then, we jump over this subcarrier and go to judge the next subcarrier.

We should note that this ordering based successive allocation (OSA) scheme is no longer globally optimal for the problem (30) with strict constraints. However, OSA scheme focuses on scheduling the subcarrier which is always beneficial to the system energy efficiency and thereby can result in a good performance. In addition, it is only of linear complexity since the problem is directly addressed in fractional manner. We call this two-step method for the energy efficiency OFDMA design as the iEE ordering based successive allocation (iEEOSA) algorithm, which is summarized in Algorithm 2, where $E E^{\prime}$ 
and $p_{l}^{\prime}$ respectively denote the system energy efficiency and the power allocation of the OSA scheme for given $\rho$.

Algorithm 2 iEE ordering based successive allocation algorithm

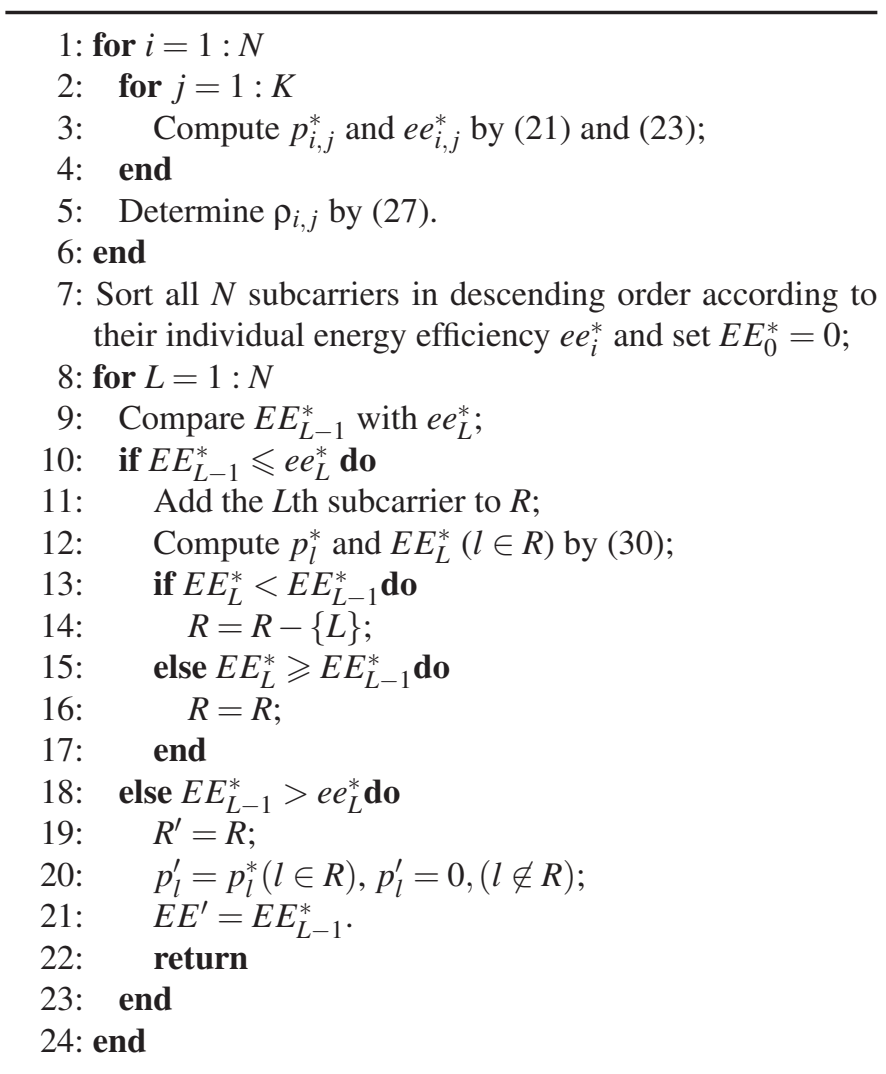

In order to get deeper understanding of the iEEOSA method, in the next, we provide a sufficient condition under which our proposed iEEOSA method is optimal for problem (8).

Theorem 4: If all users have the same signal processing power $P_{s j}$ and they are equally weighted, then the iEEOSA method is optimal for the original problem (8).

Proof: Please see Appendix D.

This theorem is due to the fact that when all users have the same weight $\omega_{j}$ and the same signal processing power $P_{s j}$, the optimal user with the highest channel gain $g_{i, j}$ is also the user with highest iEE $e e_{i, j}^{*}$ on each subcarrier, which means that the $\mathrm{iEE}$ based scheme for the subcarrier assignment and the OSA scheme for the power allocation even with constraints are both optimal according to Theorem 2 and Corollary 1, respectively. Therefore, the optimality of the iEEOSA method is guaranteed.

\section{Complexity Analysis}

In this subsection, we provide the complexity comparison between the exhaustive search method, the joint optimization method, and the iEEOSA method. For the exhaustive search method, since we also need to optimize the number of active subcarriers, then each subcarrier have $K+1$ possibilities.
TABLE I

SYSTEM PARAMETERS

\begin{tabular}{|c|c|}
\hline Parameter & Value \\
\hline Cell radius, $r$ & $1 \mathrm{~km}$ \\
\hline Subcarrier spacing, $B$ & $15 \mathrm{kHz}$ \\
\hline Maximum transmit power, $P_{\max }$ & $33 \mathrm{dBm}$ \\
\hline Minimum system date rate, $R_{\min }$ & $100 \mathrm{bps}$ \\
\hline Signal processing power of the BS, $P_{s 0}$ & $40 \mathrm{~mW}$ \\
\hline Signal processing power of the user $j, P_{s j}$ & $5-30 \mathrm{~mW}$ \\
\hline Power spectral density of thermal noise,$N_{0}$ & $-174 \mathrm{dBm} / \mathrm{Hz}$ \\
\hline Static circuit power of the BS, $P_{b}$ & $2000 \mathrm{~mW}$ \\
\hline Static circuit power of user $j, P_{u j}$ & $50 \mathrm{~mW}$ \\
\hline Efficiency of the power amplifier, $\xi$ & 0.38 \\
\hline Path loss model & Okumura-Hata \\
\hline Lognormal Shadowing & $8 \mathrm{~dB}$ \\
\hline Penetration loss & $20 \mathrm{~dB}$ \\
\hline Small scale fading model & Rayleigh fading \\
\hline
\end{tabular}

Therefore, its total complexity is about $O\left((K+1)^{N} \Omega\right)$, where $\Omega$ is complexity of given subcarrier assignment [5]. The complexity of the JO method is about $O\left(L K N 2^{\alpha}\right)$, where $L$ is the iterations for the convergence of the Dinkelbach method and $2^{\alpha}$ is the iterations of updating two dual variables [35]. For the iEEOSA method, its complexity mainly lies in computing $e e_{i, j}^{*}$ in the first step and linearly obtaining the active subcarriers set $R$ in the second step. Therefore, its total complexity is about $O(K N)$. Compared with the JO method, the complexity for the convergence of updated $q$ in the out layer and dual variables in the inner layer is reduced.

\section{Simulation Results}

In this section, we provide simulation results to demonstrate the effectiveness of the proposed resource allocation methods for the joint transmitter and receiver energy efficiency maximization. The cell of the network is hexagonal with a radius of 1000 meters, within which the users are randomly and uniformly distributed outside of the concentric circle with the radius of 100 meters. We assume that there are five users in the system and the user weights are $0.5,0.75,1,1.25$, and 1.5 , respectively. The main system parameters are from [5], [17] as listed in Table I if without specific explanation. The fixed circuit power of the BS and the user terminal is set to be $2000 \mathrm{~mW}$ and $50 \mathrm{~mW}$ as in [5] and [17], respectively. In order to characterize different types of user terminals, we assume that signal processing powers per subcarrier of different users vary from $5 \mathrm{~mW}$ to $30 \mathrm{~mW}$ and the signal processing power per subcarrier of the BS is set to be $40 \mathrm{~mW}$ as in [17], which also almost coincides with the baseband consumption level in [18]. For all simulations, 10000 channel realizations are executed.

\section{A. Convergence and Optimality of Joint Optimization (JO) Method}

Fig. 1 depicts the energy efficiency achieved with the number of iterations of the joint optimization (JO) method under small $K$ and $N$. The red dashed lines are the results of the exhaustive search method. Since only for small value, it is possible to carrier out the exhaustive search within a reasonable computation time. As seen in Fig. 1, JO method can always converge and 


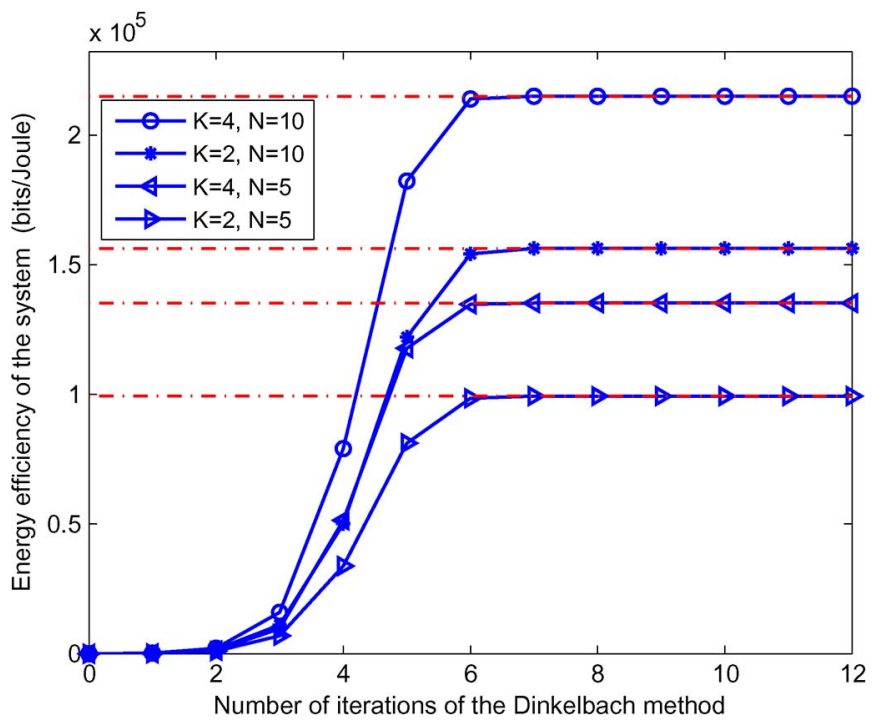

Fig. 1. The system energy efficiency achieved versus the number of the iterations using the $\mathrm{JO}$ method.

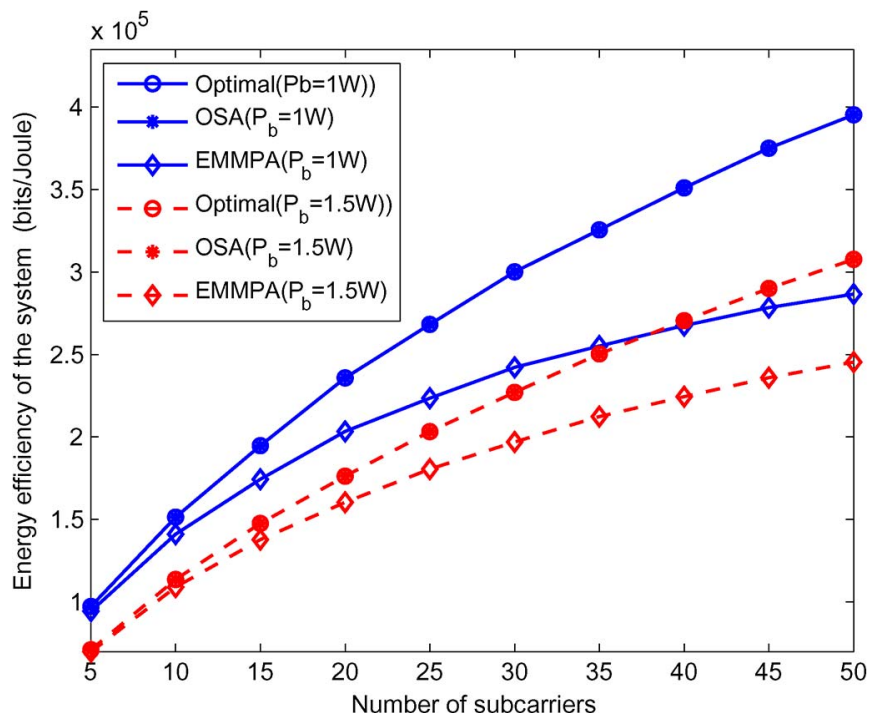

Fig. 2. The performance comparison of improved (Optimal), OSA, and EMMPA schemes under two $P_{b}$ cases $(K=5)$.

obtain the optimal solutions for all the configurations, which demonstrates the optimality of the JO method.

\section{B. Optimality of OSA Scheme for Power Allocation Without Constraints}

Fig. 2 illustrates the comparison of the OSA, the improved scheme in [27] and the EMMPA scheme [27]. The improved scheme and the EMMPA scheme are proposed to obtain the optimal and the suboptimal power allocation solutions to the problem (30) without constraints. However, the improved scheme has a quadric complexity. In order to demonstrate the optimality of the OSA for the power allocation, without loss of generality, we assume that each subcarrier is randomly assigned to a user. It can be observed that the OSA scheme is optimal and significantly outperforms the EMMPA scheme.

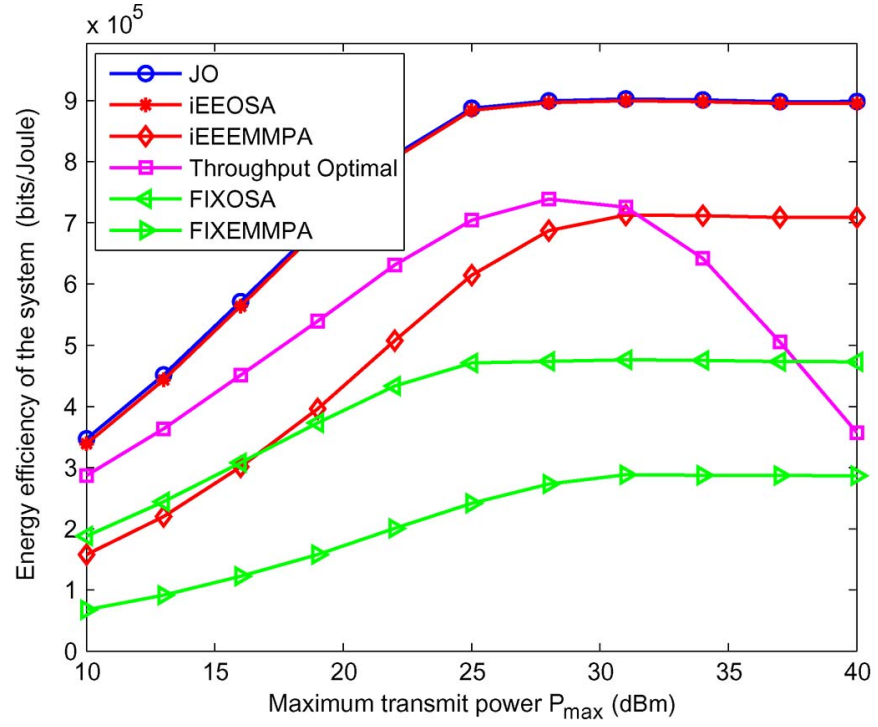

Fig. 3. The system energy efficiency versus the maximum transmit power $(K=5, N=128)$.

\section{System Energy Efficiency and Throughput Versus Maximum Transmit Power}

In Fig. 3, we compare the system energy efficiency of the following methods: 1) JO; 2) iEEOSA; 3) Throughput Optimal: the convention throughout maximization problem [22]; 4) iEEEMMPA: SEE based subcarrier assignment with EMMPA; 5) FIXOSA: Fixed subcarrier assignment with OSA; 6) FIXEMMPA: Fixed subcarrier assignment with EMMPA.

As we can observe that iEEOSA method can achieve the near-optimal performance of the optimal energy efficiency, and only in the lower power region, it suffers a slight performance loss mainly due to its non-optimality for the maximal power constraint case. It is interesting to note that in the low power regime, although the JO method and the Throughput Optimal method both increase with the transmit power, the JO method still outperforms the Throughput Optimal method, which implies that the energy efficiency maximization problem is not equivalent to the throughput maximization problem. The reason is that even with the fixed transmit power, the system power consumption may still vary depending on the number of active subcarriers. As the transmit power increases, the constraint becomes relaxed, iEEOSA almost achieve the same performance as the optimal method, which also demonstrates our theoretical analysis of constraints absent case, while the throughput optimization method results in low energy efficiency due to its overusing of the transmit power. In addition, from the comparison of iEEOSA and RAOSA, IEEEMMPA and RAEMMPA, the advantage of the proposed iEE based assignment scheme is demonstrated. Comparing JO and Throughput Optimal, we can observe the tradeoff between the energy efficiency and the system throughput.

Fig. 4 further demonstrates the effectiveness of the proposed method and the energy efficiency and the spectral efficiency tradeoff. The Throughput Optimal method always results in the highest throughput at the cost of sacrificing energy efficiency while energy efficiency oriented designs approach constants due to their conservative nature of using power. 


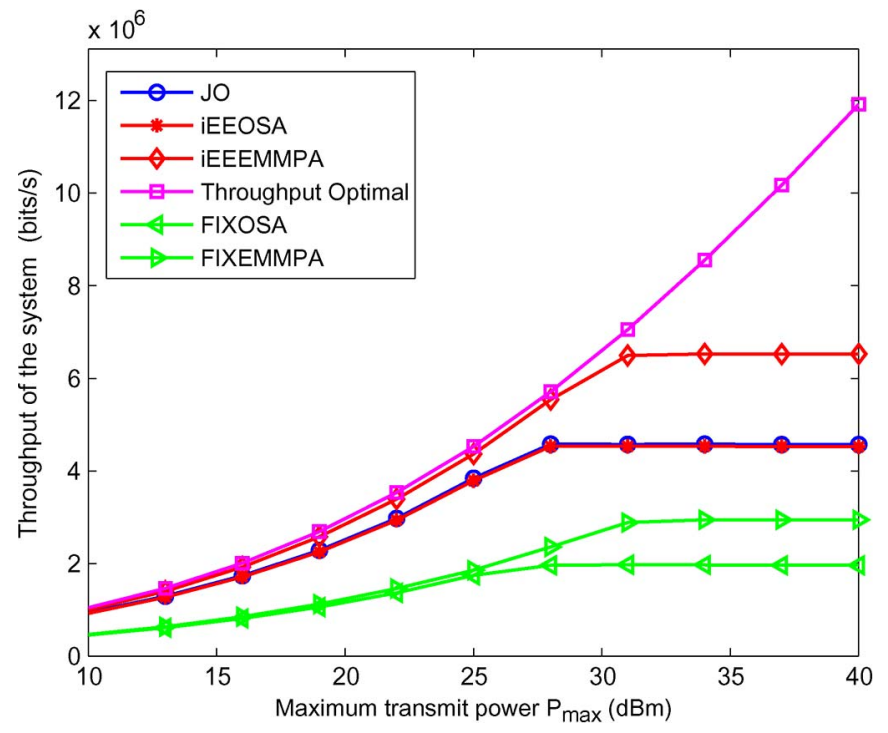

Fig. 4. The system throughput versus the maximum transmit power $(K=5$, $N=128$ ).

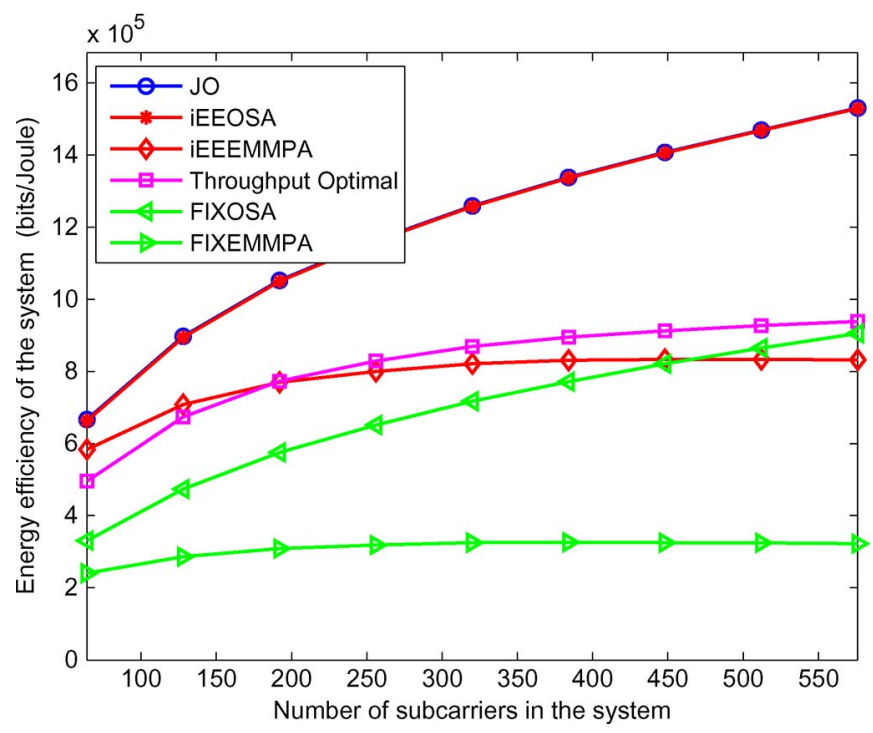

Fig. 5. The system energy efficiency versus the number of subcarriers $(K=5)$.

\section{System Energy Efficiency Versus Number of Subcarriers and Number of Users}

In Fig. 5, we compare the system energy efficiency versus number of subcarriers and the number of users for all methods mentioned in Fig. 3. It can be observed that JO, iEEOSA can increase the system energy efficiency in a large range number of subcarriers, while iEEEMMPA and FIXMMPA are very easily to make the system energy efficiency saturate since they do not optimize the number of active subcarriers.

Fig. 6 shows the energy efficiency versus the number of users. We can see that within the medium-sized number of users, iEEOSA still achieve near-optimal performance, while for large number of users, it results in a slight performance loss since there are much more possibilities for the subcarrier assignment. In addition, when the number of users is large, the efficient power allocation is not attractive due to the multiuser diversity, and this is also why iEEOSA converges with iEEEMMPA.

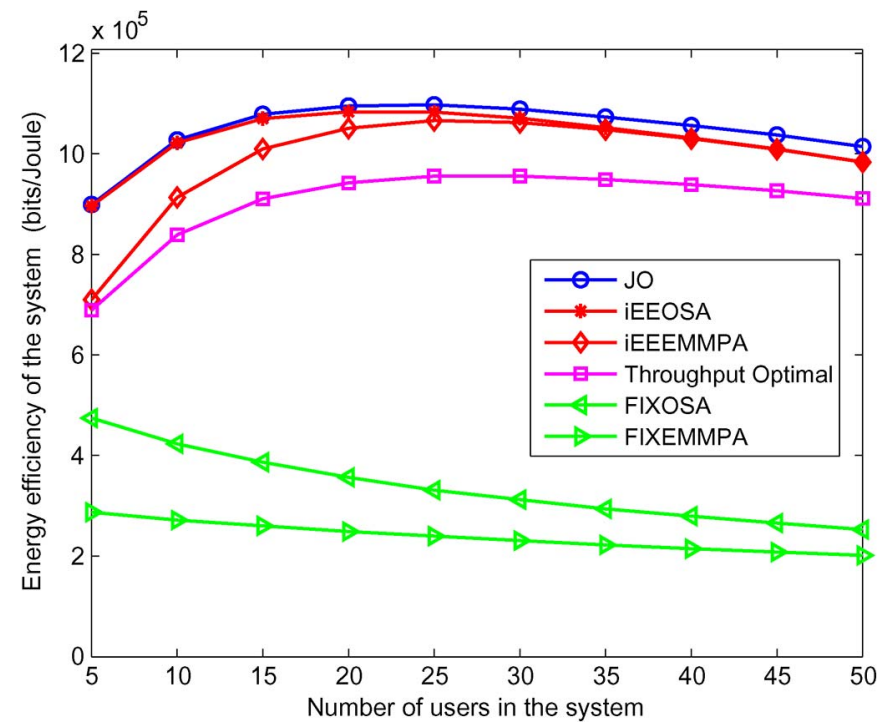

Fig. 6. The system energy efficiency versus the number of users and all the users have the same weight $(N=128)$.

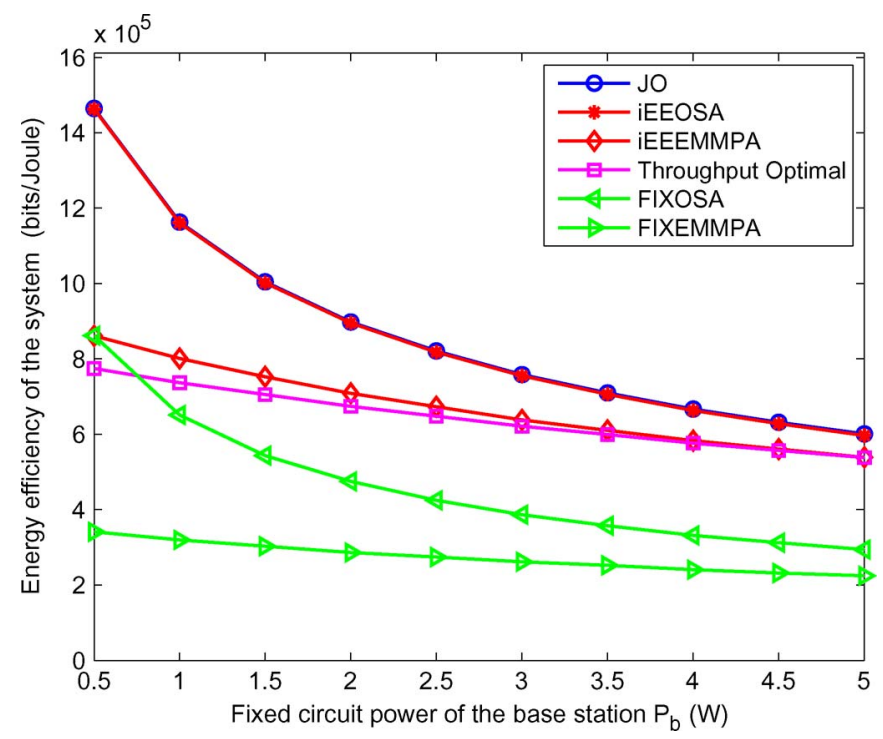

Fig. 7. The effects of the fixed circuit power of the BS on the system energy efficiency $(K=5, N=128)$.

\section{E. System Energy Efficiency Versus Fixed Power Consumption of $B S$ and Cell Radius}

The effects of the fixed power consumption $P_{b}$ of the BS on the system energy efficiency is depicted in Fig. 7. We can see that the energy efficiency of all the methods decrease with the increasing of the $P_{b}$. Additionally, it is notable that the performance gap between IEEMMPA and iEEOSA decreases as the $P_{b}$ increases. This can be readily understood since when the $P_{b}$ is larger, it becomes more dominated in the total power consumption, which weakens the performance benefits from the energy efficiency oriented power allocation.

Fig. 8 characterizes the effect of the cell radius on the energy efficiency. As expected, the energy efficiency of all the methods decreases with the increasing size of the cell due to the increasing path loss. Moreover, we notice that the performance gap between iEEMMPA and iEEOSA increases when the cell 


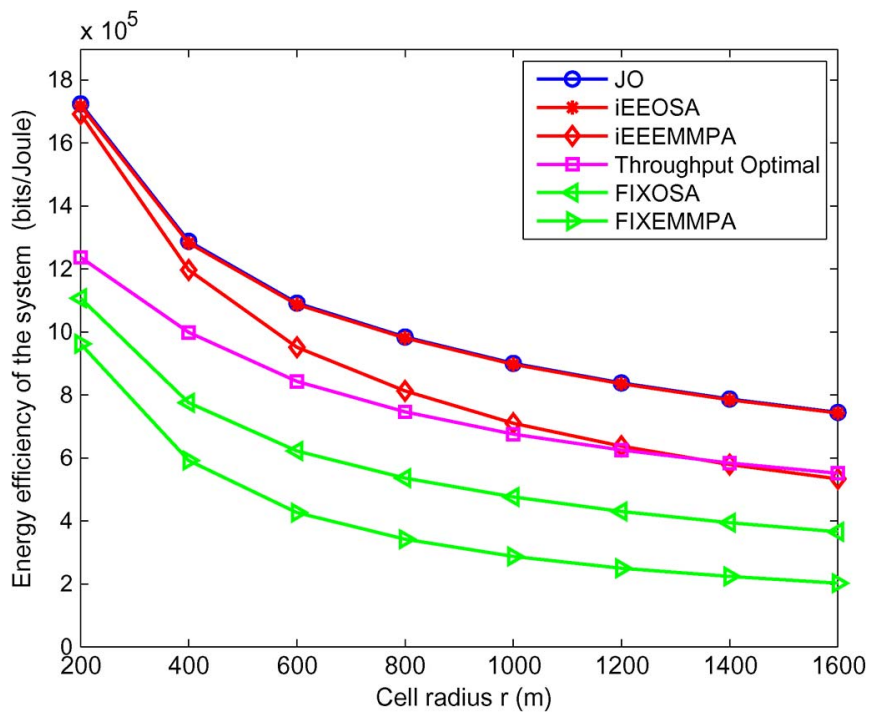

Fig. 8. The effects of the cell radius on the system energy efficiency $(K=$ $5, N=128)$.

radius increases. Because when the channel conditions of users vary in a large range, the effective power allocation plays a critical important role for energy efficiency optimization.

\section{F. Effects of Imperfect CSIT on System Energy Efficiency}

In this subsection, demonstrate the effects of imperfect CSIT on the system performance. The channel gain is given by $g_{i, j}=l_{j}\left|h_{i, j}\right|^{2}$, where $l_{j}$ represents the large-scale attenuation and $h_{i, j}$ is the small-scale fading coefficient between the BS and user $j$ on subcarrier $i$. Since the path loss and the shadowing are slowly varying random processes which both change on the order of seconds for low mobility users, the large-scale attenuation are assumed estimated perfectly [26]. Therefore, following the convention, to capture the effect of imperfect CSIT, the multipath fading CSIT between the BS and user $j$ on subcarrier $i$ can be typically modeled as [26], [36]:

$$
h_{i, j}=\widetilde{h}_{i, j}+\Delta h_{i, j}
$$

where $h_{i, j}$ and $\widetilde{h}_{i, j}$ are the actual CSIT and the estimated CSIT of user $j$ on subcarrier $i$, respectively, available to the central controller, while $\triangle h_{i, j}$ is the corresponding CSIT error. Here, $\widetilde{h}_{i, j}$ and $\triangle h_{i, j}$ are independent Gaussian random variables. Besides, $h_{i, j}, \widetilde{h}_{i, j}$ and $\triangle h_{i, j}$ have zero means and normalized variances of $1,1-\xi$, and $\xi$, respectively [26].

As observed in Fig. 9, with the increasing of the channel estimation error $\xi$, the system energy efficiency decreases, and the gap between perfect CSIT and imperfect CSIT increases. In addition, the low complexity iEEOSA method also achieves most performance of the JO method under the same channel estimation error, which further demonstrates its effectiveness for the energy-efficient resource allocation. Moreover, we can also note that as the cell size $r$ becomes larger, the performance loss caused by imperfect CSIT would be degraded. This is because although imperfect CSIT weakens the effectiveness of the resource allocation, when the channel condition is worse, the

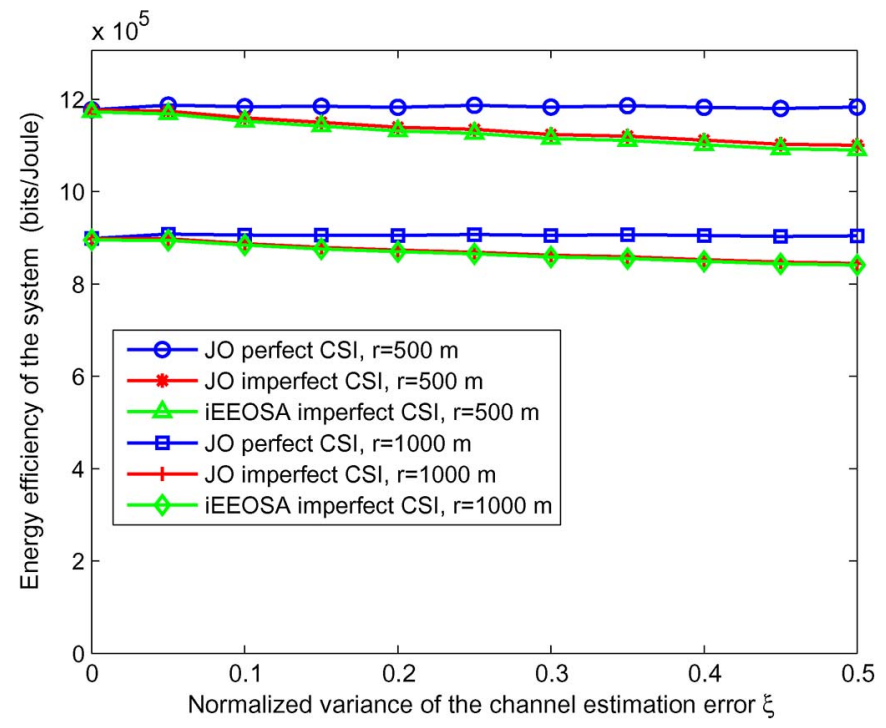

Fig. 9. The effects of the channel estimation error on the system energy efficiency $(K=5, N=128)$.

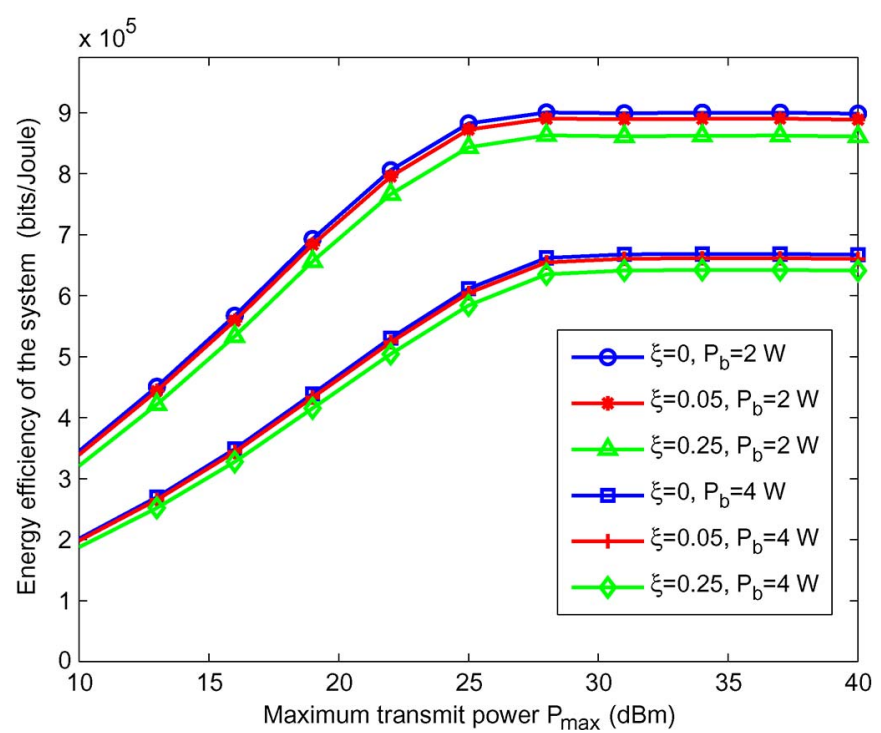

Fig. 10. The system energy efficiency versus the maximum transmit power under different channel estimation errors $(K=5, N=128)$.

energy efficiency oriented resource allocation tends to utilize more transmit power and in turn degrades the sensitivity of resource allocation strategy to imperfect CSIT.

In Fig. 10, we show the variation of the system energy efficiency with the maximum transmit power under different channel estimation errors $\xi$ and static circuit powers $P_{b}$. It is observed that the system energy efficiency loss under fixed $\xi$ will first increase and then keep constant with the increase of the maximum transmit power, which contradicts the phenomenon in throughput maximization field where the throughput loss would be compensated by increasing the transmit power. This is due to the fact that the energy efficiency oriented design makes the resource allocation more sensitive to the channel at first, and then its conservative nature of utilizing power leads the performance loss at fixed $\xi$ robust to the transmit power. Also, 
the performance loss due to imperfect CSIT is less for the BS with larger $P_{b}$, which is mainly due to the fact that larger circuit power $P_{b}$ naturally leads to higher system throughput so as to improve the energy efficiency and thus makes performance less sensitive to imperfect CSIT.

Based on the above results, we conclude that the proposed energy-efficient resource allocation can still provide insights for practical systems under imperfect CSIT except for some performance loss. In addition, the performance loss caused by imperfect CSIT can also be compensated by larger coverage and larger static circuit power of the BS.

\section{CONClusion And Future Work}

In this paper, we have investigated the joint transmitter and receiver optimization for the energy efficiency of OFDMA systems, where a practical power consumption model is established to capture the effects of active subcarriers and users on the signal processing power and the static circuit power, respectively. Then, we propose a joint optimization method to iteratively solve the energy efficiency maximization problem based on Dinkelbach transformation. To provide more insights into low complexity designs, we first introduce the individual energy efficiency of a subcarrier-user pattern and then propose an efficient two-step method. The key idea of this method is to explore the inherent relationship of the individual energy efficiency and the system energy efficiency. Numerical results show the tradeoff between the energy efficiency and the spectral efficiency, and the impacts of subcarriers and users on the system energy efficiency. Moreover, it is also found that exploiting more user diversity is not always beneficial from the perspective of system energy efficiency.

There are also several important issues unaddressed in this paper and left for future work. First, Individual data rate constraint for each user: Since the low complexity iEEOSA method first assigns subcarriers to users from the system perspective instead of each user, the quality of service of individual user can not be guaranteed. The challenge of addressing the individual QoS is how to jointly assign subcarriers and allocate the power so as to satisfy the data rate requirement while achieving the maximal system energy efficiency. Second, Nonlinear power amplifier: In practice, adopting the non-linear power amplifier may largely improve the system efficiency, but its modeling and problem solving issues still need further investigation. Third, Optimal number of users: We have shown that more users may not be helpful for the system energy efficiency and how to design the user scheduling policy is also an interesting issue. Last but not the least, Robust resource allocation: Designing practical methods for the resource allocation under imperfect channel CSIT is also very challenging [37].

\section{APPENDIX A}

\section{PROOF OF THEOREM 2}

Assume that the user $k$ satisfies the properties of Theorem 2, i.e., $\omega_{k}=\max _{j=1 \ldots, K} \omega_{j}, g_{m, k}=\max _{j=1 \ldots, K} h_{m, j}$, and $P_{s k}=\min _{j=1 \ldots, K} P_{s j}$. Denote $E E^{*}(m, j)$ as the optimal energy efficiency of assigning subcarrier $m$ to any user $j(j \neq k)$ under the transmit power constraint and the data rate constraint. Let $\hat{p}_{i, j}$ and $\hat{\rho}_{i, j}$ be the corresponding power allocation and indicator of assigning subcarrier $m$ to user $j$, respectively. Denote $E E^{*}(m, k)$ as the optimal energy efficiency of selecting the user $k$ for the subcarrier $m$ under constraints. Let $\breve{p}_{i, j}$ and $\check{\rho}_{i, j}$ be the corresponding power allocation and indicator of assigning subcarrier $m$ to user $k$, respectively. Then

$$
\begin{aligned}
& E E^{*}(m, j) \\
& =\frac{\sum_{i \neq m} \sum_{j=1}^{K} \hat{\rho}_{i, j} \omega_{j} r_{i, j}\left(\hat{p}_{i, j}\right)+\omega_{j} B \log _{2}\left(1+\frac{\hat{p}_{m, j} h_{m, j}}{N_{0} B}\right)}{\left(\sum_{i \neq m} \sum_{j=1}^{K} \hat{\rho}_{i, j} P_{i, j}\left(\hat{p}_{i, j}\right)+P_{0}\right)+\left(\frac{\hat{p}_{m, j}}{\xi}+P_{s 0}+P_{s j}\right)} \\
& \stackrel{(a)}{\leqslant} \frac{\sum_{i \neq m} \sum_{j=1}^{K} \hat{\rho}_{i, j} \omega_{j} r_{i, j}\left(\hat{p}_{i, j}\right)+\omega_{k} B \log _{2}\left(1+\frac{\hat{p}_{m, k} g_{m, k}}{N_{0} B}\right)}{\left(\sum_{i \neq m} \sum_{j=1}^{K} \hat{\rho}_{i, j} P_{i, j}\left(\hat{p}_{i, j}\right)+P_{0}\right)+\left(\frac{\hat{p}_{m, k}}{\xi}+P_{s 0}+P_{s k}\right)} \\
& \stackrel{(b)}{\leqslant} \frac{\sum_{i \neq m} \sum_{j=1}^{K} \check{\rho}_{i, j} \omega_{j} r_{i, j}\left(\check{p}_{i, j}\right)+\omega_{k} B \log _{2}\left(1+\frac{\check{p}_{m, k} g_{m, k}}{N_{0} B}\right)}{\left(\sum_{i \neq m} \sum_{j=1}^{K} \check{\rho}_{i, j} P_{i, j}\left(\breve{p}_{i, j}\right)+P_{0}\right)+\left(\frac{\check{p}_{m, k}}{\xi}+P_{s 0}+P_{s k}\right)} \\
& =E E^{*}(m, k)
\end{aligned}
$$

where the inequality $(a)$ is due to $g_{m, k} \geqslant g_{m, j}$ and $P_{s k} \leqslant P_{s j}$, and the inequality $(b)$ is because that $\breve{p}_{i, j}$ and $\check{\rho}_{i, j}$ are the optimal solutions corresponding to $E E^{*}(m, j)$.

Hence, for $\forall j(j \neq k)$, we have $E E^{*}(m, j) \leqslant E E^{*}(m, k)$. Therefore the user $k$ is the optimal user for subcarrier $m$ to achieve the maximal system energy efficiency. This completes the proof.

\section{APPENDIX B \\ PROOF OF THEOREM 3}

Denote $\hat{p}_{l}^{L}$ as the optimal power allocations corresponding to $E E_{L}^{*}$, and $p_{L}^{*}$ is the optimal power corresponding to the $L$ th subcarrier's energy efficiency $e e_{L}^{*}$.

1) If $E E_{L-1}^{*} \leqslant e e_{L}^{*}$,

$$
\begin{aligned}
E E_{L}^{*} & =\frac{\sum_{l=1}^{L} \omega_{l} r_{l}\left(\hat{p}_{l}^{L}\right)}{\sum_{l=1}^{L} P_{l}\left(\hat{p}_{l}^{L}\right)+P_{0}} \\
& =\max _{p} \frac{\sum_{l=1}^{L} \omega_{l} r_{l}\left(p_{l}\right)}{\sum_{l=1}^{L} P_{l}\left(p_{l}\right)+P_{0}} \\
& \stackrel{(a)}{\geqslant} \frac{\sum_{l=1}^{L-1} \omega_{l} r_{l}\left(\hat{p}_{l}^{L}\right)+\omega_{L} r_{L}\left(p_{L}^{*}\right)}{\sum_{l=1}^{L-1} P_{l}\left(\hat{p}_{l}^{L}\right)+P_{0}+P_{L}\left(p_{L}^{*}\right)} \\
& \geqslant \min \left\{\frac{\sum_{l=1}^{L-1} \omega_{l} r_{l}\left(\hat{p}_{l}^{L}\right)}{\sum_{l=1}^{L-1} P_{l}\left(\hat{p}_{l}^{L}\right)+P_{0}}, \frac{\omega_{L} r_{L}\left(p_{L}^{*}\right)}{P_{L}\left(p_{L}^{*}\right)}\right\} \\
& =\min \left\{E E_{L-1}^{*}, e e_{L}^{*}\right\} \\
& =E E_{L-1}^{*}
\end{aligned}
$$


In this case, we know that adding the $L$ th subcarrier can obviously increase the system energy efficiency. On the other hand,

$$
\begin{aligned}
E E_{L}^{*} & =\max _{p} \frac{\sum_{l=1}^{L} \omega_{l} r_{l}\left(p_{l}\right)}{\sum_{l=1}^{L} P_{l}\left(p_{l}\right)+P_{0}} \\
& =\frac{\sum_{l=1}^{L-1} \omega_{l} r_{l}\left(\hat{p}_{l}^{L}\right)+\omega_{L} r_{L}\left(\hat{p}_{L}^{L}\right)}{\sum_{l=1}^{L-1} P_{l}\left(\hat{p}_{l}^{L}\right)+P_{0}+P_{L}\left(\hat{p}_{L}^{L}\right)} \\
& \leqslant \max \left\{\frac{\sum_{l=1}^{L-1} \omega_{l} r_{l}\left(\hat{p}_{l}^{L}\right)}{\sum_{l=1}^{L-1} P_{l}\left(\hat{p}_{l}^{L}\right)+P_{0}}, \frac{\omega_{L} r_{L}\left(\hat{p}_{L}^{L}\right)}{P_{L}\left(\hat{p}_{L}^{L}\right)}\right\} \\
& \leqslant \max \left\{\frac{\sum_{l=1}^{L-1} \omega_{l} r_{l}\left(\hat{p}_{l}^{L-1}\right)}{\sum_{l=1}^{L-1} P_{l}\left(\hat{p}_{l}^{L-1}\right)+P_{0}}, \frac{\omega_{L} r_{L}\left(p_{L}^{*}\right)}{P_{L}\left(p_{L}^{*}\right)}\right\} \\
& =\max \left\{E E_{L-1}^{*}, e e_{L}^{*}\right\} \\
& =e e_{L}^{*} .
\end{aligned}
$$

Based on (33) and (34), we have

$$
E E_{L-1}^{*} \leqslant E E_{L}^{*} \leqslant e e_{L}^{*}
$$

2) If $E E_{L-1}^{*}>e e_{L}^{*}$, we can prove $E E_{L-1}^{*}>E E_{L}^{*}>e e_{L}^{*}$ by a similar argument in the case 1). In this case, adding the $L$ th subcarrier cannot increase the system energy efficiency. This completes the proof.

\section{APPENDIX C \\ ProOF OF COROLlaRY 1}

We first prove that the optimal powers $\left\{\hat{p}_{l}^{L}\right\}$ allocated to subcarriers in the set $R$ will be positive after adding the $L$ th subcarrier to $R$ by the iEE ordering based scheme. Assume $\hat{p}_{l}^{L}=0$ for some $l \in\{1,2 \ldots, L\}$, which means that the $l$ th subcarrier should be removed from $R$ since $R$ is the set of users with positive powers. Then according to Theorem 3 we have

$$
E E_{L-1}^{*} \leqslant E E_{L}^{*} \leqslant e e_{L}^{*}, \text { when } E E_{L-1}^{*} \leqslant e e_{L}^{*}
$$

Since the subcarriers are added to $R$ in the descending order, we have $e e_{L}^{*} \leqslant e e_{l}^{*}$. Then, we have $E E_{L}^{*} \leqslant e e_{L}^{*} \leqslant e e_{l}^{*}$. Based on Theorem 3 , the $l$ th subcarrier should be added to $R$ and allocated with positive power, which contradicts to the assumption that the $l$ th subcarrier should be removed from $R$.

In the next, we prove that the energy efficiency based on the optimal subcarriers set $R^{o p t}$ is higher than that based on arbitrary set $\hat{R}$ with positive power allocation. For a set $R, E E_{R}^{*}$ denotes the optimal energy efficiency based on $R$.

Let $R^{o p t} \triangleq\left\{1, \cdots, L_{R^{o p t}}\right\}$. Applying the OSA scheme in Section IV-C over $\hat{R}$, we can find the optimal set $\hat{R}^{o p t}$. Assume $\hat{R}^{o p t} \triangleq\left\{l_{1}, \cdots, l_{K}\right\}$, where $l_{1}, \ldots, l_{K}$ are the indices of the corresponding $\left\{e e_{l_{K}}\right\}_{k=1}^{K}$ in descending order. Then $E E_{\hat{R}}^{*} \leqslant E E_{\hat{R}^{o p t}}^{*} \leqslant$ $e e_{l_{K}}^{*}$ by $(35)$. Note that $e e_{l_{K}}^{*} \leqslant e e_{l_{K}-1}^{*} \leqslant \cdots \leqslant e e_{1}^{*}$. By Theorem 3 and (35), we can add $\left(l_{K}-1\right)$ to the set $\hat{R}^{o p t}$ if $\left(l_{K}-1\right) \notin \hat{R}^{o p t}$ and obtain

$$
E E_{\hat{R}}^{*} \leqslant E E_{\hat{R}^{o p t}}^{*} \leqslant E E_{\hat{R}^{o p t} \cup\left\{l_{K}-1\right\}}^{*} \leqslant e e_{l_{K}-1}^{*} \leqslant e e_{l_{K}-2}^{*} \leqslant \cdots \leqslant e e_{1}^{*} .
$$

If $\left(l_{K}-1\right) \in \hat{R}^{\text {opt }}$, we jump over $\left(l_{K}-1\right)$ and consider $\left(l_{K}-2\right)$. Define

$$
\hat{R}_{1}^{\text {opt }}= \begin{cases}\hat{R}^{\text {opt }} \cup\left\{l_{K}-1\right\}, & \text { if }\left(l_{K}-1\right) \notin \hat{R}^{o p t} ; \\ \hat{R}^{\text {opt }}, & \text { otherwise. }\end{cases}
$$

If $\left(l_{K}-2\right) \notin \hat{R}_{1}^{o p t}$, we add $\left(l_{K}-2\right)$ to $\hat{R}_{1}^{o p t}$ and obtain

$$
\begin{aligned}
E E_{\hat{R}}^{*} \leqslant E E_{\hat{R}^{o p t}}^{*} \leqslant E E_{\hat{R}_{1}^{o p t}}^{*} \leqslant E E_{\hat{R}_{1}^{o p t}}^{*} & \cup\left\{l_{K}-2\right\} \\
& \leqslant e e_{l_{K}-2}^{*} \leqslant \cdots \leqslant e e_{1}^{*} .
\end{aligned}
$$

If $\left(l_{K}-2\right) \in \hat{R}_{1}^{o p t}$, we jump over $\left(l_{K}-2\right)$ and consider $\left(l_{K}-3\right)$. For $l=2, \cdots, l_{K}-1$, define

$$
\hat{R}_{l}^{o p t}= \begin{cases}\hat{R}_{l-1}^{o p t} \cup\left\{l_{K}-l\right\}, & \text { if }\left(l_{K}-l\right) \notin \hat{R}_{l-1}^{o p t} ; \\ \hat{R}_{l-1}^{o p t}, & \text { otherwise. }\end{cases}
$$

Following the similar procedure, we can successively check if $\left(l_{K}-l\right) \in \hat{R}_{l-1}^{o p t}$ for $l=3, \cdots,\left(l_{K}-1\right)$, and finally obtain

$$
E E_{\hat{R}}^{*} \leqslant E E_{\hat{R}^{o p t}}^{*} \leqslant E E_{\hat{R}_{1}^{o p t}}^{*} \leqslant E E_{\hat{R}_{2}^{o p t}}^{*} \leqslant \cdots \leqslant E E_{\hat{R}_{K^{-1}}^{o p t}}^{*} .
$$

Note that $\hat{R}_{l_{K}-1}^{o p t}=\left\{1, \cdots, l_{K}\right\}$. Since the optimal set $R^{o p t} \triangleq$ $\left\{1, \cdots, L_{R^{o p t}}\right\}$, we have $E E_{\hat{R}_{K^{-1}}^{\text {opt }}}^{*} \leqslant E E_{R^{\text {opt }}}^{*}$ by Theorem 3. Therefore we have $E E_{\hat{R}}^{*} \leqslant E E_{\hat{R}_{l_{K}-1}^{o p t}}^{*} \leqslant E E_{R^{o p t}}^{*}$ and conclude that $R^{o p t}$ is the optimal set. This completes the proof.

\section{APPENDIX D}

\section{PROOF OF THEOREM 4}

If all the users have the same signal processing power $p_{s j}$ and are equally weighted i.e., $P_{s j}=P_{s k}$ and $\omega_{j}=\omega_{k}$ for $\forall j \neq k$, then the condition $P_{s k}=\min _{j=1, \ldots, K} P_{s j}$ and $\omega_{k}=\max _{j=1, \ldots, K} \omega_{j}$ are satisfied for each subcarrier. According to Theorem 2, the optimal user for the subcarrier $m$ should satisfy the condition $g_{m, k}=$ $\max _{j=1, \ldots, K} g_{m, j}$. On the other hand, in the iEE based subcarrier assignment scheme, we choose the user with the highest $e e_{i, j}^{*}$ as the optimal user. From (23), by taking the first derivative of $e e_{i, j}^{*}$ with respect to $g_{i, j}$, we can get

$$
\frac{d e e_{i, j}^{*}}{d g_{i, j}}=\frac{\omega_{j} \xi B^{2} N_{0} \ln 2}{\left(B N_{0}+p_{i, j}^{*} g_{i, j} \ln 2\right)^{2}}>0 .
$$

It implies that $e e_{i, j}^{*}$ strictly increases with $g_{i, j}$. Then $e e_{m, k}^{*}=$ $\max _{j=1, \ldots, K} e e_{m, j}^{*}$ holds with $g_{m, k}=\max _{j=1, \ldots, K} g_{m, j}$, which implies that the iEE based subcarrier assignment scheme is optimal. Under this condition, the optimality of the OSA scheme for the power allocation can be easily proved by a similar proof of Corollary 1. 


\section{REFERENCES}

[1] J. Wu, S. Rangan, and H. Zhang, Green Communications: Theoretical Fundamentals, Algorithms and Applications. Boca Raton, FL, USA: CRC Press, 2012.

[2] G. Y. Li et al., "Energy-efficient wireless communications: Tutorial, survey, open issues," IEEE Wireless Commun., vol. 18, no. 6, pp. 28-35, Dec. 2011.

[3] G. Miao, N. Himayat, Y. Li, and D. Bormann, "Energy efficient design in wireless OFDMA," in Proc. IEEE ICC, 2008, pp. 3307-3312.

[4] G. Miao, N. Himayat, G. Y. Li, and S. Talwar, "Low-complexity energyefficient scheduling for uplink OFDMA," IEEE Trans. Commun., vol. 60, no. 1, pp. 112-120, Jan. 2012.

[5] C. Xiong, G. Li, S. Zhang, Y. Chen, and S. Xu, "Energy-efficient resource allocation in OFDMA networks," IEEE Trans. Commun., vol. 60, no. 12, pp. 3767-3778, Dec. 2012.

[6] X. Xiao, X. Tao, and J. Lu, "QoS-aware energy-efficient radio resource scheduling in multi-user OFDMA systems," IEEE Commun. Lett., vol. 17, no. 1, pp. 75-78, Jan. 2013.

[7] Q. Shi et al., "On the energy efficiency optimality of OFDMA for SISOOFDM downlink system," IEEE Commun. Lett., vol. 17, no. 3, pp. 541544, Mar. 2013.

[8] W. Cheng, X. Zhang, and H. Zhang, "Joint spectrum and power efficiencies optimization for statistical QoS provisionings over SISO/MIMO wireless networks," IEEE J. Sel. Areas Commun., vol. 31, no. 5, pp. $903-$ 915, May 2013.

[9] C. Xiong, G. Y. Li, S. Zhang, Y. Chen, and S. Xu, "Energy- and spectralefficiency tradeoff in downlink OFDMA networks," IEEE Trans. Wireless Commun., vol. 10, no. 11, pp. 3874-3886, Nov. 2011

[10] R. S. Prabhu and B. Daneshrad, "An energy-efficient water-filling algorithm for OFDM systems," in Proc. IEEE ICC, 2010, pp. 1-5.

[11] D. W. K. Ng, E. S. Lo, and R. Schober, "Energy-efficient resource allocation in multi-cell OFDMA systems with limited backhaul capacity," IEEE Trans. Wireless Commun., vol. 11, no. 10, pp. 3618-3631, Oct. 2012

[12] M. Gruber et al., "EARTH: Energy aware radio and network technologies," in Proc. IEEE PIMRC, 2009, pp. 1-5.

[13] G. Auer et al., "D2. 3: Energy efficiency analysis of the reference systems, areas of improvements and target breakdown," EARTH NFSO-ICT247733, 2010. [Online]. Available: http://ec.europa.eu/ information_society/apps/projects/logos/3/247733/080/deliverables/001_ EARTHWP2D23v2.pdf

[14] H. Kim and G. De Veciana, "Leveraging dynamic spare capacity in wireless systems to conserve mobile terminals' energy," IEEE/ACM Trans. Netw., vol. 18, no. 3, pp. 802-815, Jun. 2010.

[15] F.-S. Chu, K.-C. Chen, and G. Fettweis, "Green resource allocation to minimize receiving energy in OFDMA cellular systems," IEEE Commun. Lett., vol. 16, no. 3, pp. 372-374, Mar. 2012.

[16] S. Luo, R. Zhang, and T. J. Lim, "Joint transmitter and receiver energy minimization in multiuser OFDM systems," arXiv preprint arXiv:1312.6743, 2013

[17] Z. Xu et al., "Energy-efficient configuration of spatial and frequency resources in MIMO-OFDMA systems," IEEE Trans. Commun., vol. 61, no. 2, pp. 564-575, Feb. 2013.

[18] S. Zhang, Y. Chen, and S. Xu, "Joint bandwidth-power allocation for energy efficient transmission in multi-user systems," in Proc. IEEE GLOBECOM, 2010, pp. 1400-1405.

[19] D. W. K. Ng, E. S. Lo, and R. Schober, "Energy-efficient resource allocation in OFDMA systems with hybrid energy harvesting base station," IEEE Trans. Wireless Commun., vol. 12, no. 7, pp. 3412-3427, Jul. 2013.

[20] D. W. K. Ng, E. S. Lo, and R. Schober, "Wireless information and power transfer: Energy efficiency optimization in OFDMA systems," IEEE Trans. Wireless Commun., vol. 12, no. 12, pp. 6352-6370, Dec. 2013

[21] D. W. K. Ng, E. S. Lo, and R. Schober, "Energy-efficient resource allocation for secure OFDMA systems," IEEE Trans. Veh. Technol., vol. 61, no. 6, pp. 2572-2585, Jul. 2012

[22] M. Tao, Y.-C. Liang, and F. Zhang, "Resource allocation for delay differentiated traffic in multiuser OFDM systems," IEEE Trans. Wireless Commun., vol. 7, no. 6, pp. 2190-2201, Jun. 2008.

[23] H. S. Kim and B. Daneshrad, "Energy-constrained link adaptation for MIMO OFDM wireless communication systems," IEEE Trans. Wireless Commun., vol. 9, no. 9, pp. 2820-2832, Sep. 2010.

[24] S. Cui, A. J. Goldsmith, and A. Bahai, "Energy-efficiency of MIMO and cooperative MIMO techniques in sensor networks," IEEE J. Sel. Areas Commun., vol. 22, no. 6, pp. 1089-1098, Aug. 2004
[25] G. Miao and A. Vastberg, "Energy efficiency in the wideband regime," IEEE Trans. Wireless Commun., vol. 12, no. 8, pp. 4102-4109, Aug. 2013.

[26] D. W. K. Ng, E. S. Lo, and R. Schober, "Energy-efficient resource allocation in OFDMA systems with large numbers of base station antennas," IEEE Trans. Wireless Commun., vol. 11, no. 9, pp. 3292-3304, Sep. 2012.

[27] G. Miao, "Energy-efficient uplink multi-user MIMO," IEEE Trans. Wireless Commun., vol. 12, no. 5, pp. 2302-2313, May 2013.

[28] Linear Amplifier. [Online]. Available: http://en.wikipedia.org/wiki/ Linear_amplifier

[29] G. Auer et al., "How much energy is needed to run a wireless network?" IEEE Wireless Commun., vol. 18, no. 5, pp. 40-49, Oct. 2011.

[30] W. Dinkelbach, "On nonlinear fractional programming," Manage. Sci. vol. 13, no. 7, pp. 492-498, Mar. 1967.

[31] S. Boyd and L. Vandenberghe, Convex Optimization. Cambridge, U.K.: Cambridge Univ. Press, 2004.

[32] W. Yu and R. Lui, "Dual methods for nonconvex spectrum optimization of multicarrier systems," IEEE Trans. Commun., vol. 54, no. 7, pp. 1310 1322, Jul. 2006.

[33] G. Miao, N. Himayat, and G. Y. Li, "Energy-efficient link adaptation in frequency-selective channels," IEEE Trans. Commun., vol. 58, no. 2, pp. 545-554, Feb. 2010.

[34] J. Mao, G. Xie, J. Gao, and Y. Liu, "Energy efficiency optimization for OFDM-based cognitive radio systems: A water-filling factor aided search method," IEEE Trans. Wireless Commun., vol. 12, no. 5, pp. 2366-2375, May 2013.

[35] W. Dang, M. Tao, H. Mu, and J. Huang, "Subcarrier-pair based resource allocation for cooperative multi-relay OFDM systems," IEEE Trans. Wireless Commun., vol. 9, no. 5, pp. 1640-1649, May 2010.

[36] C. Hao and B. Clerckx, "Imperfect and unmatched CSIT is still useful for the frequency correlated MISO broadcast channel," in Proc. IEEE ICC 2013, pp. 3181-3186.

[37] B. Clerckx, H. Lee, Y.-J. Hong, and G. Kim, "A practical cooperative multicell MIMO-OFDMA network based on rank coordination," IEEE Trans. Wireless Commun., vol. 12, no. 4, pp. 1481-1491, Apr. 2013.

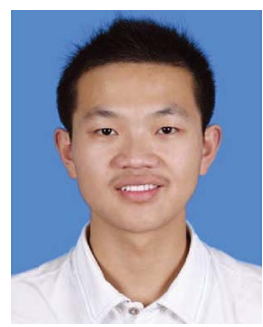

Qingqing Wu (S'13) received the B.S. degree in electronic engineering from South China University of Technology, Guangzhou, China, in 2012. He is currently working toward the Ph.D. degree with the Network Coding and Transmission Laboratory, Shanghai Jiao Tong University, Shanghai, China. His current research interests include green radio, energy-efficient optimization, and wireless information and power transfer

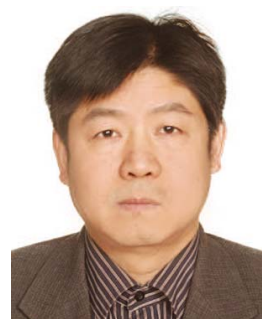

Wen Chen (M'03-SM'11) received the B.S. and M.S. degrees from Wuhan University, Wuhan, China, in 1990 and 1993, respectively, and the Ph.D. degree from the University of Electro-Communications, Tokyo, Japan, in 1999. He was a Researcher at the Japan Society for the Promotion of Science from 1999 through 2001. In 2001, he joined the University of Alberta, Edmonton, AB, Canada, starting as a Postdoctoral Fellow with the Information Research Laboratory and continuing as a Research Associate with the Department of Electrical and Computer Engineering. Since 2006, he has been a Full Professor at the Department of Electronic Engineering, Shanghai Jiao Tong University, Shanghai, China, where he is also the Director of the Institute for Signal Processing and Systems. Since 2014. he has served as the Dean of School of Electronic Engineering and Automation, Guilin University of Electronic Technology, Guilin, China. His interests cover network coding, cooperative communications, cognitive radio, and MIMO-OFDM systems. In this area, he has published 57 papers in IEEE journals and more than 100 papers in IEEE conferences. 


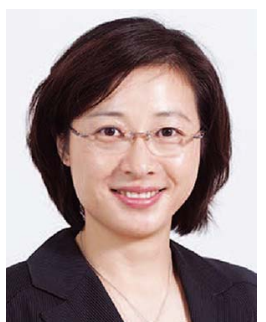

Meixia Tao (S'00-M'04-SM'10) received the B.S. degree in electronic engineering from Fudan University, Shanghai, China, in 1999 and the Ph.D. degree in electrical and electronic engineering from the Hong Kong University of Science and Technology, Clear Water Bay, Hong Kong, in 2003. She is currently a Professor with the Department of Electronic Engineering, Shanghai Jiao Tong University, Shanghai, China. Prior to that, she was a Member of Professional Staff at the Hong Kong Applied Science and Technology Research Institute during 2003-2004 and a Teaching Fellow then an Assistant Professor at the Department of Electrical and Computer Engineering, National University of Singapore, Singapore. from 2004 to 2007. Her current research interests include cooperative communications, wireless resource allocation, MIMO techniques, and physical layer security.

Dr. Tao has served as the TPC Chair of IEEE/CIC ICCC 2014 and is serving as the Symposium Co-Chair of IEEE ICC 2015. She is an Editor of the IEEE TRANSACTIONS ON COMMUNICATIONS and the IEEE WIRELESS COMMUNiCATIONS LETTERS. She was on the Editorial Board of the IEEE TRANSACTIONS ON WIRELESS COMMUNICATIONS from 2007 to 2011 and the IEEE COMMUNICATIONS LETTERS from 2009 to 2012. She also served as a Guest Editor of the IEEE COMMUNICATIONS MAGAZINE with feature topic on LTE-Advanced and 4G Wireless Communications in 2012 and a Guest Editor of EURASIP Journal on Wireless Communications and Networking with special issue on Physical Layer Network Coding for Wireless Cooperative Networks in 2010.

He was the recipient of the IEEE Heinrich Hertz Award for Best Communications Letters in 2013, the IEEE ComSoc Asia-Pacific Outstanding Young Researcher Award in 2009, and the International Conference on Wireless Communications and Signal Processing Best Paper Award in 2012.

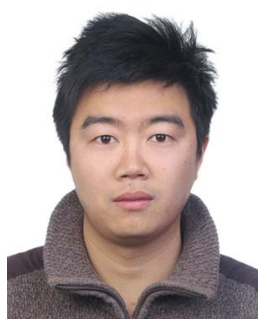

Jun Li (M’09) received Ph.D. degree in electronic engineering from Shanghai Jiao Tong University, Shanghai, China, in 2009. From January 2009 to June 2009, he was a Research Scientist with the Department of Research and Innovation, Alcatel Lucent Shanghai Bell. From June 2009 to April 2012, he was a Postdoctoral Fellow at the School of Electrical Engineering and Telecommunications, University of New South Wales, Sydney, Australia. Since April 2012, he has been a Research Fellow at the School of Electrical Engineering, The University of Sydney, Sydney. His research interests include network information theory, channel coding theory, wireless network coding and cooperative communications. Dr. Li served as the Technical Program Committee Member for several international conferences such as APCC2009, APCC2010, VTC2011 (Spring), ICC2011, TENCON2012, APCC2013, VTC2014 (Fall), and ICC2014.

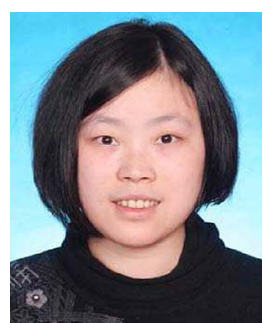

Hongying Tang received the B.S. degree in electronic engineering from the University of Electronic Science and Technology of China, Chengdu, China, in 2007 and the M.S. degree in electronic engineering from Shanghai Jiao Tong University, Shanghai, China, in 2009. She is currently working toward the Ph.D. degree with the Network Coding and Transmission Laboratory, Department of Electronic Engineering, Shanghai Jiao Tong University. Her current research interests include cooperative networks and multiple-input-multiple-output techniques.

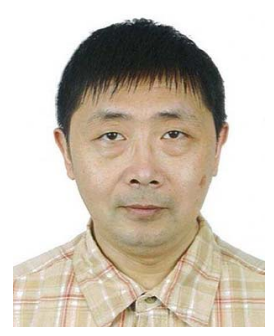

Jinsong Wu (SM'11) received the Ph.D. degree in electrical engineering from Queen's University, Kingston, ON, Canada. He is currently with the Department of Electrical Engineering, Universidad de Chile, Santiago, Chile. He was the leading Editor and a co-author of the comprehensive book Green Communications: Theoretical Fundamentals, Algorithms, and Application (CRC Press, 2012) Dr. Wu is the Founder and Founding Chair of the Technical Committee on Green Communications and Computing (TCGCC), IEEE Communications Society, which was established in 2011 as an official Technical Subcommittee (TSCGCC) and elevated as TCGCC in 2013. He is an Associate Editor of the IEEE COMmUniCATIONS SuRveys and TUtorials, an Associate Editor of the IEEE Systems Journal, and an Associate Editor of IEEE ACCESS. He is an Area Editor of the incoming IEEE Journal SELECTED AREAS ON COMMUNICATIONS (JSAC) Series on Green Communications and Networking starting in 2015. He is the Founder and Series Editor on Green Communication and Computing networks of the IEEE COMMUNICATIONS MAGAZINE. He has served as a co-leading Guest Editor of Special Issue on Green Communications, Computing, and Systems in the IEEE SYSTEMS Journal and an Associate Editor of Special Section on Big Data for Green Communications and Computing in IEEE ACCESS. 